Une approche territoriale et anticipatrice

pour une transhumance apaisée à

la frontière entre le Togo et le Burkina Faso

Synthèse
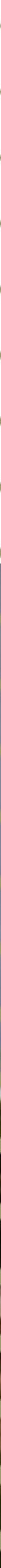



\section{Une approche territoriale et anticipatrice pour une transhumance apaisée à la frontière entre le Togo et le Burkina Faso}

Synthèse

\section{Auteurs}

Jean-Michel Sourisseau

Centre de coopération internationale en recherche agronomique pour le développement

Véronique Ancey

Organisation des Nations Unies pour I'alimentation et l'agriculture 
Citer comme suit:

Sourisseau, J.-M. et Ancey, V. 2021. Une approche territoriale et anticipatrice pour une transhumance apaisée à la frontière entre le Togo et le Burkina Faso - Synthèse. Rome, FAO. https://doi.org/10.4060/cb7925fr

Les appellations employées dans ce produit d'information et la présentation des données qui y figurent n'impliquent de la part de l'Organisation des Nations Unies pour l'alimentation et l'agriculture (FAO) aucune prise de position quant au statut juridique ou au stade de développement des pays, territoires, villes ou zones ou de leurs autorités, ni quant au tracé de leurs frontières ou limites. Le fait qu'une société ou qu'un produit manufacturé, breveté ou non, soit mentionné ne signifie pas que la FAO approuve ou recommande ladite société ou ledit produit de préférence à d'autres sociétés ou produits analogues qui ne sont pas cités.

Les opinions exprimées dans ce produit d'information sont celles du/des auteur(s) et ne reflètent pas nécessairement les vues ou les politiques de la FAO.

ISBN 978-92-5-135444-5

(C) FAO, 2021

\section{(๑) $\odot$}

Certains droits réservés. Cette œuvre est mise à la disposition du public selon les termes de la Licence Creative Commons Attribution-Pas d'Utilisation Commerciale-Partage dans les Mêmes Conditions 3.0 Organisations Intergouvernementales (CC BY NC SA 3.0 IGO; https://creativecommons.org/licenses/by-nc-sa/3.0/igo/legalcode.fr).

Selon les termes de cette licence, cette œuvre peut être copiée, diffusée et adaptée à des fins non commerciales, sous réserve que la source soit mentionnée. Lorsque l'œuvre est utilisée, rien ne doit laisser entendre que la FAO cautionne tels ou tels organisation, produit ou service. L'utilisation du logo de la FAO n'est pas autorisée. Si l'œuvre est adaptée, le produit de cette adaptation doit être diffusé sous la même licence Creative Commons ou sous une licence équivalente. Si l'œuvre est traduite, la traduction doit obligatoirement être accompagnée de la mention de la source ainsi que de la clause de non-responsabilité suivante: «La traduction n'a pas été réalisée par l'Organisation des Nations Unies pour I'alimentation et l'agriculture (FAO). La FAO n'est pas responsable du contenu ni de l'exactitude de la traduction. L'édition originale française est celle qui fait foi.»

Tout litige relatif à la présente licence ne pouvant être résolu à l'amiable sera réglé par voie de médiation et d'arbitrage tel que décrit à l'Article 8 de la licence, sauf indication contraire contenue dans le présent document. Les règles de médiation applicables seront celles de l'Organisation mondiale de la propriété intellectuelle (http://www.wipo.int/amc/fr/mediation/ rules) et tout arbitrage sera mené conformément au Règlement d'arbitrage de la Commission des Nations Unies pour le droit commercial international (CNUDCI).

Matériel attribué à des tiers. II incombe aux utilisateurs souhaitant réutiliser des informations ou autres éléments contenus dans cette œuvre qui y sont attribués à un tiers, tels que des tableaux, des figures ou des images, de déterminer si une autorisation est requise pour leur réutilisation et d'obtenir le cas échéant la permission de l'ayant-droit. Toute action qui serait engagée à la suite d'une utilisation non autorisée d'un élément de l'œuvre sur lequel une tierce partie détient des droits ne pourrait l'être qu'à l'encontre de l'utilisateur.

Ventes, droits et licences. Les produits d'information de la FAO sont disponibles sur le site web de la FAO (www.fao.org/ publications) et peuvent être obtenus sur demande adressée par courriel à: publications-sales@fao.org. Les demandes visant un usage commercial doivent être soumises à: www.fao.org/contact-us/licence-request. Les questions relatives aux droits et aux licences doivent être adressées à: copyright@fao.org. 


\section{Table des matières}

Remerciements iv

A propos de ce document $v$

1. JUSTIFICATION, OBJECTIFS ET MÉTHODE

2. ÉLÉMENTS DE DIAGNOSTIC ET PRINCIPALES LEÇONS

2.1 LE POIDS DE LA CROISSANCE DÉMOGRAPHIQUE SUR LES DYNAMIQUES TERRITORIALES 7

2.2 LA NÉCESSAIRE MAIS PROBLÉMATIQUE DIVERSIFICATION ÉCONOMIQUE

2.3 APERÇU SUR LES CONDITIONS DE VIE: DES TERRITOIRES MARGINALISÉS DANS LEUR
ESPACE NATIONAL

2.4 DES ENQUÊTES TÉMOIGNANT DE REPRÉSENTATIONS CONTRASTÉES SUR L'AVENIR DU TERRITOIRE $\quad 14$

3. ENSEIGNEMENTS DES ATELIERS D’ANTICIPATION: AIDE À LA DÉCISION ET PASSAGE À L'ACTION 17

3.1 LA CO-ÉLABORATION PARTICIPATIVE DE SCÉNARIOS

3.2 APERÇU DE QUELQUES RECOMMANDATIONS POUR L'ACTION 21

3.3 LES PERSPECTIVES OFFERTES POUR LE TERRITOIRE PAR LE TRAVAIL PARTICIPATIF RÉALISÉ 22

4. QUELQUES PERSPECTIVES POUR LE TERRITOIRE ET LE PASTORALISME 25

4.1 RECONNAÎTRE ET VALORISER LES RESSOURCES TERRITORIALES PAR DES
STRATÉGIES RÉSOLUMENT TRANSFRONTALIÈRES

4.2 INSCRIRE LA MOBILITÉ PASTORALE DANS CETTE STRATÉGIE TRANSFRONTALIÈRE 26

4.3 ENTRETENIR UNE DYNAMIQUE COLLECTIVE TRANSFRONTALIÈRE ET NON
CORPORATISTE POUR RENFORCER LA RÉFLEXION STRATÉGIQUE

5. BIBLIOGRAPHIE INDICATIVE 29 


\section{Remerciements}

Ce document a été préparé par Jean-Michel Sourisseau et Véronique Ancey, sur la base des résultats produits par l'équipe technique CIRAD-ISRA dans le cadre de l'étude de prospective territoriale pour une transhumance apaisée à la frontière du Burkina Faso et du Togo, réalisée en 2020 et 2021 dans le cadre d'une Lettre d'agrément CIRAD-FAO.

Cette synthèse bénéficie des apports de plusieurs chercheurs et partenaires à différents stades de l'étude. Au Burkina Faso, Boubacar Maiga et Jérôme Thiombiano pour le Réseau de communication sur le pastoralisme (RECOPA), au Togo Paul Kanfitine pour l'ONG Communication pour un développement durable (CDD) ont supervisé la mise en œuvre des enquêtes et des ateliers. Le Bureau régional au Bénin de la Coopération suisse a fourni un appui financier complémentaire à l'organisation des ateliers. Le consortium des partenaires a également bénéficié des données et de l'expertise de Gabriel Sangli, de l'Université de Ouagadougou au Burkina Faso et, au Togo, de l'Institut togolais de recherche agronomique (ITRA), de l'ONG Gestion de l'environnement et valorisation des produits agropastoraux et forestiers (GEVAPAF) à Dapaong, du point focal sur la transhumance du Ministère de l'agriculture et de l'élevage, du Ministère de la planification du développement et de la coopération et de l'Université de Kara.

La rédaction du rapport et l'animation des ateliers de prospective en 2019 et 2020 ont mobilisé au CIRAD Jean-François Bélières, Robin Bourgeois, Christian Corniaux, Camille Jahel, Guillaume Soullier, Ibra Touré; à l'Institut sénégalais de recherche agricole (ISRA), Astou Diao Camara.

La version définitive de ce document a été révisée par Alberto Bigi.

Les auteurs remercient chaleureusement les chercheurs et personnes ressources de chacune de ces institutions, les familles rencontrées au cours des enquêtes et bien entendu les «experts du territoire à la frontière du Togo et du Burkina Faso»; sans l'équipe dynamique qu'ils ont formée durant les trois semaines des ateliers, rien n’aurait été possible. 


\section{À propos de ce document}

Les crises affectant le pastoralisme dans la région subsaharienne depuis quelques années s'inscrivent dans des conflits territoriaux.

La branche Productions animales et ressources génétiques (NSAG) de la FAO informe le dialogue public sur l'élevage et le pastoralisme par des résultats scientifiques sur l'économie, l'environnement et les contextes sociaux des systèmes pastoraux, pour favoriser leur soutien politique dans un contexte où nouvelles urgences, inégalités et compétitions accrues sur les ressources mettent en question les habitats et les conditions de vie des pasteurs.

Ainsi, la convention de recherche signée par la FAO et le Centre de coopération internationale en recherche agronomique pour le développement (CIRAD), dont est issu ce document, visait à compléter et enrichir les initiatives locales pour une transhumance transfrontalière apaisée et, au-delà, les réponses régionales aux défis de la mobilité des animaux et des éleveurs. Le travail présenté ici en synthèse a rempli deux objectifs principaux. La méthode innovante de l'approche anticipatrice et territoriale a offert des espaces de concertation originaux et inédits à des «experts» locaux représentatifs de plusieurs facettes de la population du territoire transfrontalier, qui sont désormais formés à utiliser cet outil. La démarche complète (analyse des données existantes, enquête auprès de plus de 500 ménages, entretiens et trois ateliers en groupe pérenne) a abouti à des recommandations collectives pour un développement territorial - donc multisectoriel - renforçant les places et rôles de la transhumance et favorisant sa coexistence apaisée avec les autres secteurs d'activité dans les territoires.

Pour en savoir plus, le rapport complet de l'étude est disponible en ligne: Vers une transhumance apaisée à la frontière entre le Togo et le Burkina Faso? Perspectives d'une approche territoriale et anticipatrice 



\section{Justification, objectifs et méthode}

Cette recherche-action fait suite à la crise pastorale en 2017 au Sahel, qui s'est caractérisée davantage par des encombrements et des tensions transfrontalières Sahelcôtes que par l'ampleur des déficits fourragers saisonniers sur les parcours septentrionaux des éleveurs transhumants. Le contexte transfrontalier entre le Sahel et les pays côtiers s'est tendu à cause de l'instabilité politique au Sahel (et en particulier dans l'est du Burkina Faso) et de la crainte des pays côtiers (et notamment le Togo) d'une contagion sur leur territoire de cette instabilité. Les contraintes et les risques liés à la mobilité pastorale saisonnière s'accroissent, alors même que les stratégies pastorales, optimisant la variabilité des ressources naturelles, s'inscrivent dans les dynamiques territoriales démographiques, agricoles et politiques. Depuis fin 2017, les restrictions au passage des frontières et l'application de règlements limitant la mobilité des pasteurs ont été un facteur de risque supplémentaire, aggravant la situation pastorale dans le Sahel (texte de position de la FAO et du Programme alimentaire mondial (PAM), rencontre de Niamey, 2018). Les tensions autour de la transhumance ont entraîné des dégâts dans les cultures, l'abattage sauvage d'animaux et des morts humaines, notamment au Nigéria, Bénin et Ghana. Mais ces événements ponctuels s'inscrivent aussi dans les transformations structurelles des territoires induites par les politiques nationales de décentralisation, agricoles et économiques. Faute d'extension aboutie des services et des équipements publics dans les régions périphériques, le risque est grand de voir s'aggraver la déstructuration d'espaces ruraux en cours de densification. Enfin, l'absence de mise en œuvre d'une politique inclusive régionale au sujet de la transhumance et des outils de régulation des marchés a des conséquences lourdes sur la vie économique et sociale.

Des textes communautaires et des négociations locales bilatérales existent: le protocole de la Communauté économique des États de l'Afrique de l'Ouest (CEDEAO) sur la libre circulation des personnes, des biens et des services est une pierre angulaire de l'intégration régionale, qui doit être protégée; la CEDEAO a en outre approuvé en 2003 un règlement (C/REG.3/01/03) sur l'application des règlements de la transhumance entre États membres. Mais, malgré des initiatives locales de comités transfrontaliers de la transhumance créés pour gérer les zones d'accueil et les routes de transhumance, ces règlements ne se traduisent pas par des actions à la hauteur des enjeux. Il manque des programmes régionaux pour systématiser le développement des zones pastorales et des infrastructures pour la transhumance.

Face à ces constats, et conjointement à la poursuite des initiatives régionales pour le renforcement du dialogue et des soutiens au pastoralisme, l'hypothèse est faite que le double recours à des approches territoriales et à l'anticipation peut être adapté à certains défis du pastoralisme transfrontalier. En effet, la prospective territoriale offre deux avantages:

- Elle permet de replacer les mobilités animales et les questions de gestion des ressources à une échelle plus large que celle des seuls acteurs du pastoralisme, en s'intéressant à la place de la transhumance dans le développement des territoires plutôt qu'à la dynamique du seul secteur de l'élevage mobile. 
- Elle permet de s'extirper des vives tensions actuelles et de dépassionner les débats; en se projetant dans le futur, en libérant l'imagination autant que possible face à la menace sécuritaire, elle ouvre sur de nouvelles perspectives.

Le territoire d'étude se situe au nord du Togo et à l'est du Burkina Faso. Il illustre parfaitement les enjeux économiques et politiques des échanges entre un sud côtier jusqu'ici plutôt préservé des tensions terroristes et un Sahel qui ne parvient pas à retrouver stabilité et paix, vivant une situation sécuritaire en constante dégradation depuis le début des années 2010.

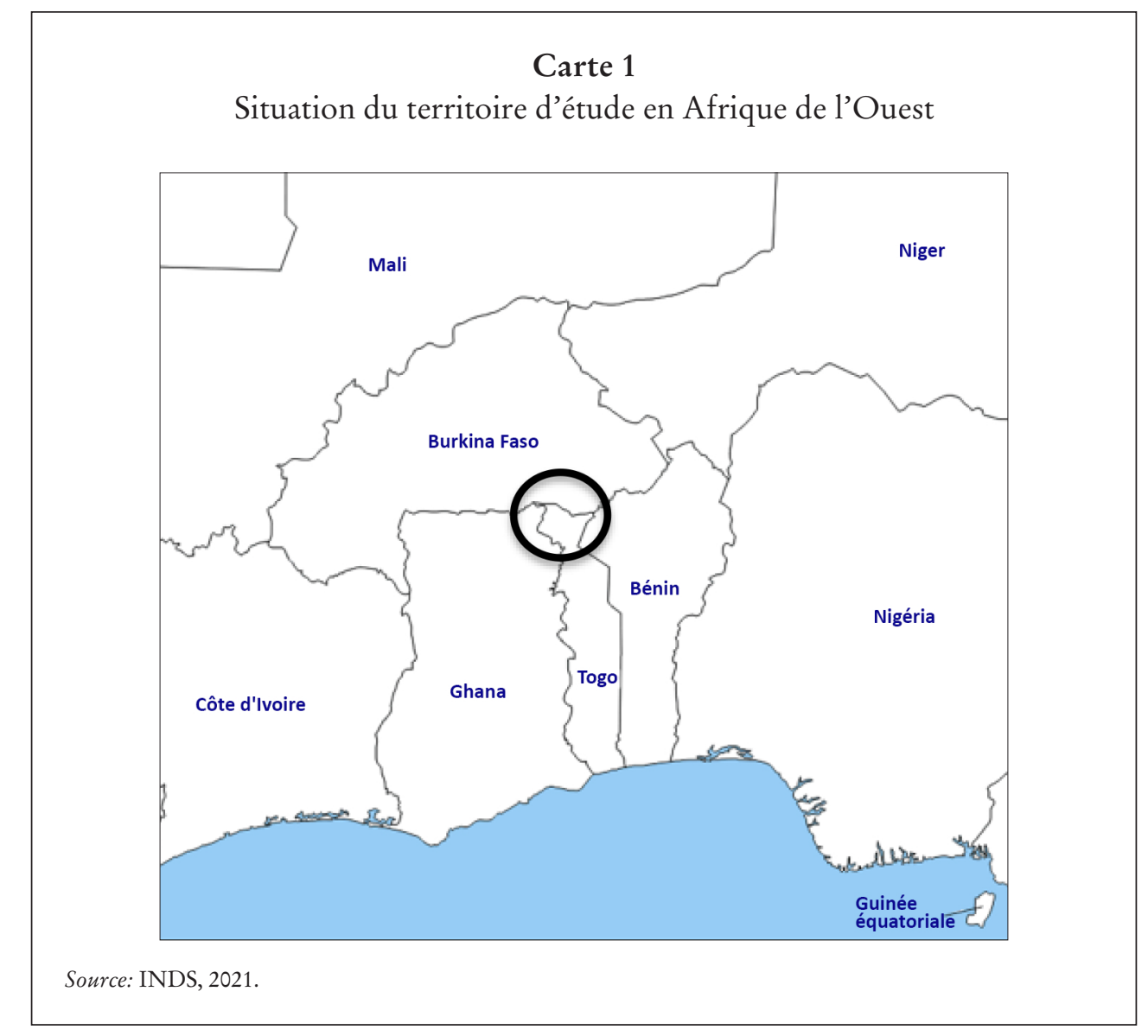

La principale richesse du Togo est son port permettant le commerce via mer et terre avec ses voisins. Ainsi, l'accès au Burkina Faso est vital pour l'économie nationale, et l'activité à la frontière au poste de Cinkassé témoigne du dynamisme et de l'ampleur des flux marchands entre la côte et le Sahel via la route internationale.

De l'autre côté de la frontière, les échanges avec le Togo sont tout aussi stratégiques. Les zones frontalières sont en effet, malgré des ressources naturelles prometteuses, parmi les plus défavorisées du Burkina Faso, et sont particulièrement touchées par l'insécurité. Dans ce contexte, elles se tournent vers le sud plus accessible aujourd'hui que la plupart des autres zones actives de leur pays. 


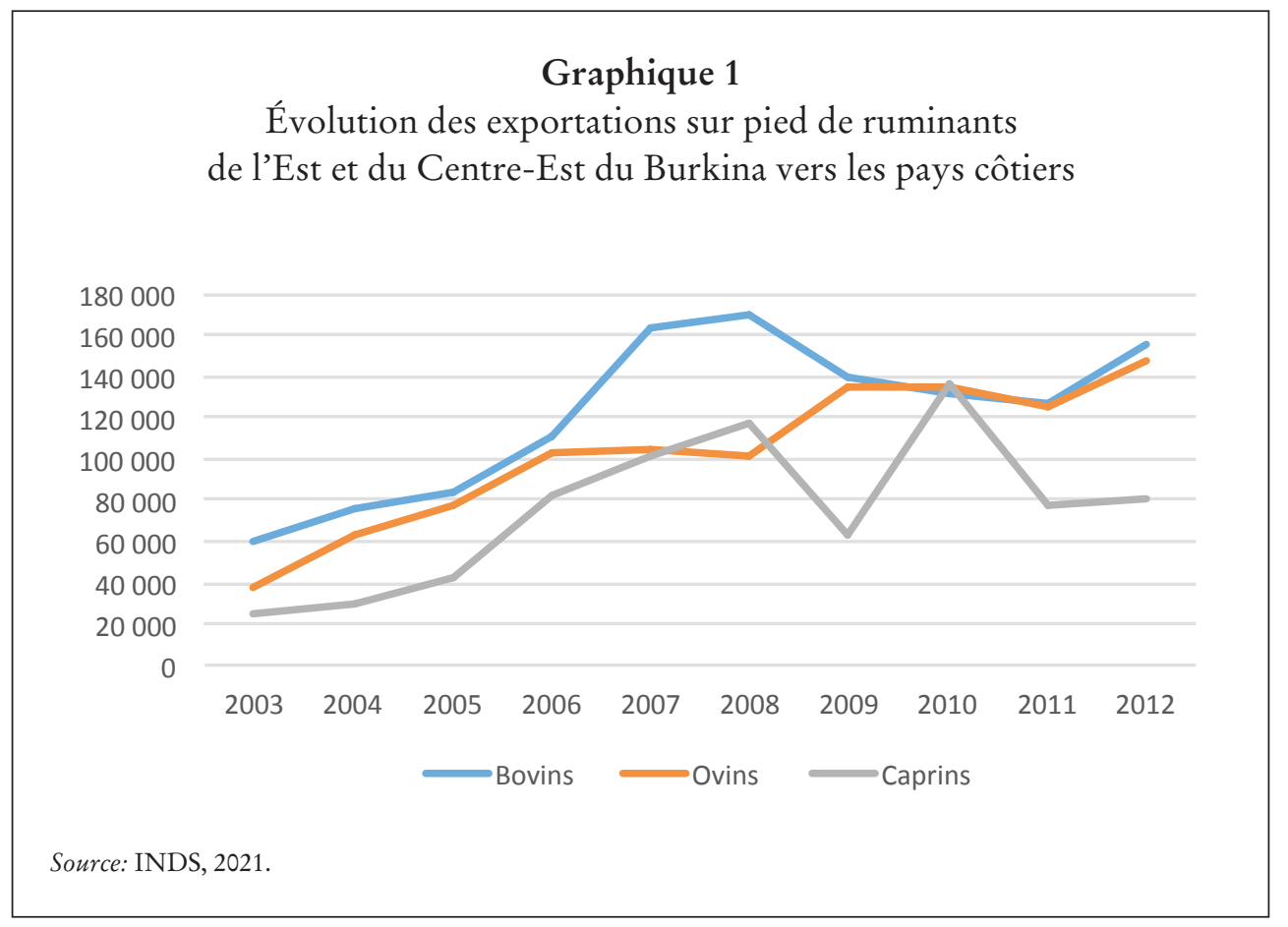

Ces flux induisent des activités conséquentes, en plus des taxes bénéficiant d'abord au pays et, moins significativement, à la zone frontalière. La frontière est aussi un point de repère pour les pasteurs déplaçant leurs animaux à la recherche de ressources fourragères et vers les marchés.

Le flux transfrontalier d'animaux comprend des exportations en expansion qui contribuent à la fourniture de bœufs de labour pour les agriculteurs et à l'apport en protéines animales aux habitants de la côte. Certains troupeaux transhument aussi en sens inverse, passant du Togo au Burkina Faso durant l'hivernage. Ces déplacements contribuent aux dynamiques commerciales, activités et richesses induites des échanges transfrontaliers et bénéficient à tout le territoire, au Togo comme au Burkina Faso.

Le choix de cette zone frontalière se justifie donc d'abord par cette activité pastorale inscrite dans un territoire transfrontalier dynamique. Il se justifie aussi parce que le Togo, par rapport à ses voisins, a résolument choisi de mettre en place des politiques de gestion des flux d'animaux. Son objectif est de réguler plutôt que de réduire la circulation, de prévenir les conflits avec les agriculteurs via un encadrement des déplacements plutôt que de mettre en place des mécanismes répressifs.

On assiste ainsi, de fait, à des mouvements contraires en termes de territorialisation transfrontalière. D'une part, la menace terroriste au Burkina Faso et la multiplication des conflits entre agriculteurs et éleveurs - amplifiés par l'instrumentalisation de certains évènements - ont tendance à durcir les conditions de passage de la frontière et à limiter les échanges. D'autre part, les communautés de part et d'autre de la frontière ont, en ces temps difficiles, besoin de davantage commercer, et plus largement de renforcer les liens sociaux, de «faire territoire» par-delà les limites administratives. La transhumance occupe une place particulière, à la fois intégratrice et source de tension, dans ces recompositions territoriales, ce qui renforce encore l'intérêt générique de la zone pour penser le pastoralisme transfrontalier. 
Un autre intérêt de l'étude, mais qui rend aussi son exécution difficile, réside dans le fait que la zone fait territoire alors même qu'elle est soumise à un découpage administratif qui contraint fortement ses logiques territoriales. Le territoire couvre en effet trois entités administratives principales de part et d'autre de la frontière. Au Burkina, il s'agit des provinces de la Kompienga, dans la région Est, et du Koulpélogo, dans la région Centre-Est. Au Togo, il s'agit de la région des savanes, qui occupe toute la partie nord du pays. Ces premiers éléments permettent de comprendre la différence radicale entre l'approche territoriale et prospective, intégrant la transhumance, et une approche sectorielle centrée sur la spatialisation des données d'élevage. Dans le contexte politiquement délicat décrit ici, l'approche territoriale et prospective fournit un diagnostic d'ensemble sur les ressources, les contraintes et les dynamiques auxquelles participe l'élevage transhumant. Elle permet de restituer les perceptions sociales du présent dans leur diversité, voire leur antagonisme, et de construire des images collectives des futurs possibles.

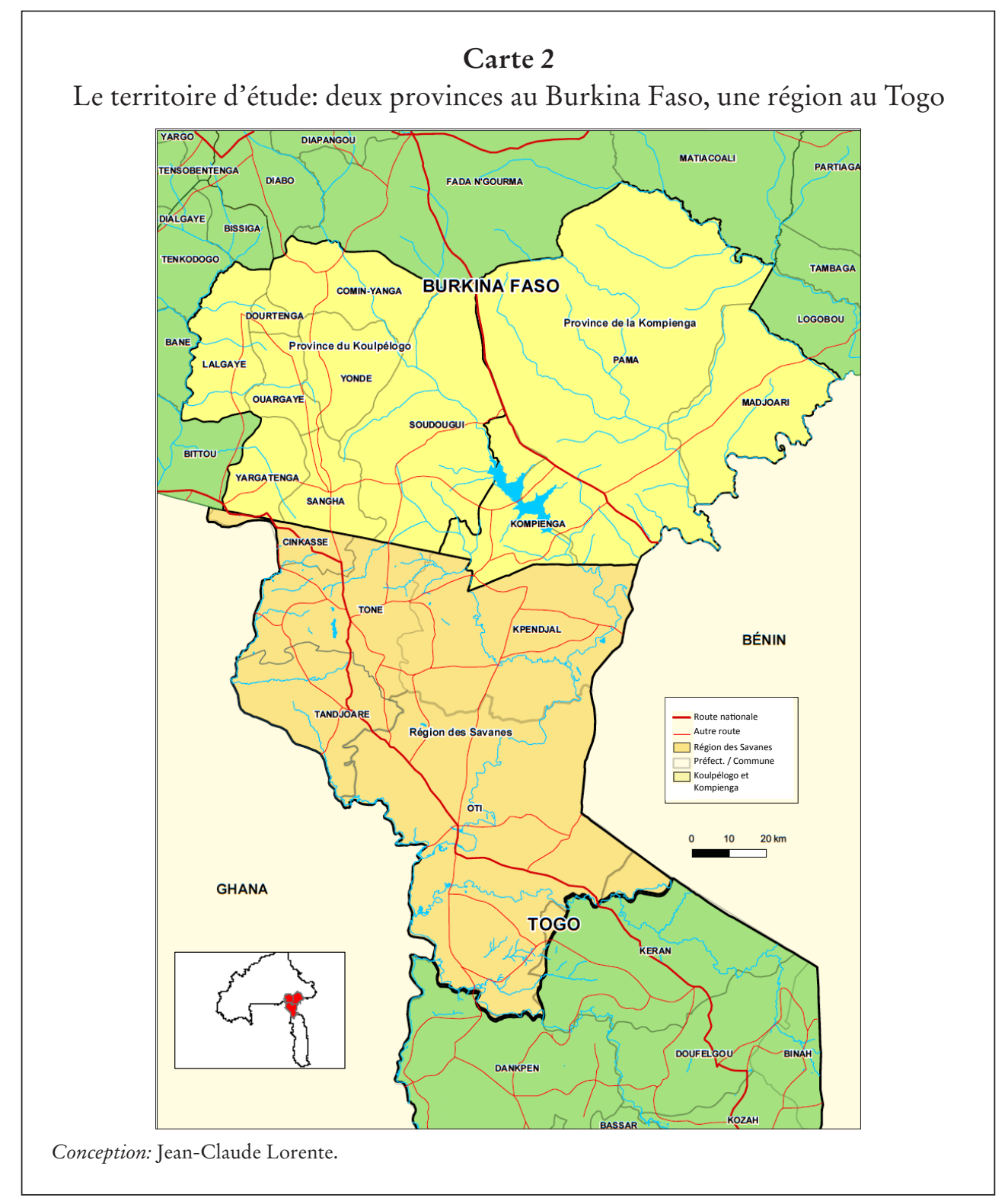


La province du Koulpélogo comprend une commune urbaine (Ouargaye), sept communes rurales (Comin-Yanga, Dourtenga, Lalgaye, Sangha, Soudigui, Yargatenga, Yondé) et 184 villages pour une superficie de $2492 \mathrm{~km}^{2}$. La province de la Kompienga occupe $7280 \mathrm{~km}^{2}$. Elle comprend une commune urbaine, Pama, comprenant elle-même 14 villages, et deux communes rurales, Kompienga (comprenant 17 villages) et Madjoari (comprenant huit villages).

La région des Savanes occupe $8470 \mathrm{~km}^{2}$. Elle est divisée en sept préfectures (à l'ouest, Cinkassé, Tone, Tandjouaré; à l'est, Kpendjal, Kpendjal-Ouest, Oti, Oti-Sud), avec un sous-découpage récent ayant donné lieu à la création de 16 communes.

Du fait de politiques de décentralisation timides, souvent inabouties et peinant à être pleinement mises en œuvre, les deux provinces burkinabé et la région des $\mathrm{Sa}$ vanes sont incitées, dans leur réflexion stratégique et sa mise en œuvre, à se tourner vers leurs capitales nationales respectives. Dans l'organisation administrative, les schémas de développement sont écrits et structurés autour des instances régionales, mais sur la base d'une réflexion, d'objectifs et d'indicateurs nationaux. Il s'agit là d'une force contraire à la territorialisation, qu'il ne faut pas négliger. Cette gouvernance centralisatrice laisse aujourd'hui peu de marge de manœuvre aux acteurs des communes et des préfectures, mais aussi des régions, pour nouer des alliances et conduire des politiques dans leur espace régional transfrontalier.

Ces contraintes organisationnelles et administratives se traduisent aussi, très concrètement, par la grande difficulté à trouver des données homogènes, cohérentes et synchrones dans les différentes entités administratives composant l'espace transfrontalier étudié. La reconstruction d'une trajectoire territoriale est ainsi une véritable gageure.

L'étude confirme la pertinence de penser la frontière comme un lien territorial dont il faudrait dépasser les ruptures de gouvernance, et valide le fait que ces trois entités administratives font territoire. Elle confirme que des perspectives d'une gouvernance plus intégrée de ce territoire sont réelles, en particulier pour apaiser les échanges et en premier lieu la transhumance. Mais elle confirme aussi que l'organisation actuelle des États et de leur administration a tendance à minimiser ces perspectives et à privilégier le repli sur soi.

Une des originalités de l'étude est la diversité des approches proposées, chacune constituant une étude dans l'étude, l'ensemble formant un tout cohérent.

Ainsi un diagnostic territorial dynamique a été construit, qui alimente la prospective participative proprement dite. Ce diagnostic résulte d'un assemblage de trois grandes sous-études:

- Une exploration bibliographique du territoire a compilé des données jusqu'alors éparses pour tenter de reconstituer grossièrement le passé et le présent du territoire. Cette vision dynamique présente de nombreuses limites, la complexité de fonctionnement d'ensemble d'un territoire ne pouvant être capturée par l'analyse des seuls jeux de données statistiques.

- La trajectoire démographique des trois entités administratives du territoire transfrontalier a été retracée et étendue à des projections de la population selon différentes hypothèses aux horizons 2035 et 2040. Ces projections démographiques sont ensuite utilisées pour estimer les besoins en terres, en infrastructures et en emplois du territoire à l'horizon 2035. 
- Deux enquêtes ont été réalisées pour compléter la revue de la littérature et des bases de données par des données de première main. Ainsi, 568 personnes $^{1}$ et 61 personnes ressources, de profils très divers ${ }^{2}$, ont été interrogées de part et d'autre de la frontière sur leurs représentations du passé et du présent, et leur projection à l'horizon 2035. Le focus a davantage été mis ici sur la transhumance et sur sa perception par les enquêtés. L'objectif est de ne pas s'en tenir aux visions des acteurs de la transhumance, mais aussi de ceux et celles qui ont un rapport plus distant avec elle.

La prospective proprement dite a été mise en ouvre au travers de deux ateliers de co-élaboration de scénarios. Ces ateliers ont réuni 21 experts et expertes (11 du Togo et $10 \mathrm{du}$ Burkina), animés par une équipe de sept personnes. Les experts et expertes sont des membres d'associations et de collectifs (RECOPA, Coordination des organisations féminines du Gourma, Cadre de concertation des éleveurs de l'Est, Centrale d'autopromotion paysanne (CAP) de Sikbaog, ONG U-CMECS (Union des caisses mutuelles d'épargne et de crédit des Savanes), l'ANOPER, APIDEV, des exploitants agricoles, des formateurs, des commerçantes en céréales, des élus locaux (adjoint au maire d'Ouargaye, maire de la commune de Tone2), des fonctionnaires (gendarmes à la retraite et en activité, directeur provincial de l'éducation de la Kompienga, directeur provincial des ressources animales et halieutiques de Koulpélogo, directeurs d'écoles, trésorier de la préfecture de Cinkassé, points focaux «transhumance» aux Ministères de l'agriculture du Togo et du Bénin), des enseignants chercheurs, un planificateur-aménagiste et un journaliste.

Partant d'une sélection des grandes variables de changement du territoire, le groupe a sélectionné huit forces motrices. Puis, en imaginant des états du futur de ces forces et en les combinant, le groupe a ébauché 15 synopsis, réduits à huit images du territoire en 2035, intégrant la place que pourrait occuper la transhumance dans ces futurs.

Enfin, un troisième atelier, valorisant les représentations du futur ainsi construites, et réunissant le même groupe d'experts, a produit des premières recommandations, ciblant la gouvernance locale et la coopération transfrontalière pour une transhumance apaisée, dans le contexte actuel des politiques publiques nationales et de la coopération régionale.

La logique d'organisation du rapport peut être synthétisée à travers le schéma suivant.

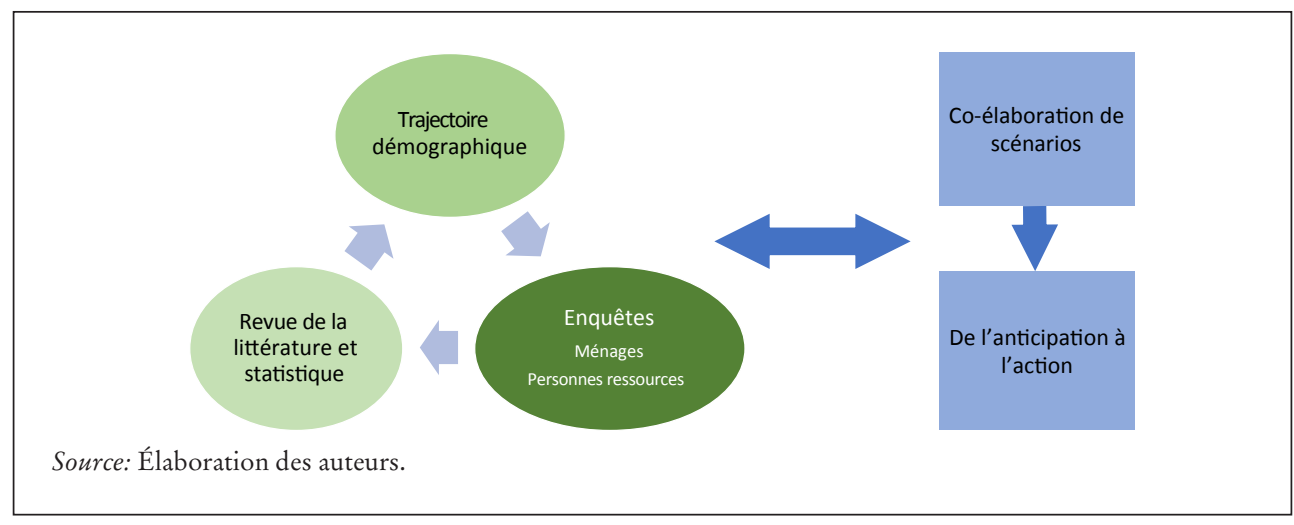

239 en tant que chef d'exploitation agricole, 207 femmes et 122 jeunes au sein de ces 239 exploitations.

230 personnes au Togo et 31 au Burkina, 18 sont plutôt expertes du secteur de l'agriculture et de l'élevage, 9 du secteur du commerce, 7 de l'éducation et de la santé, 27 sont des élus ou représentants de l'administration. 


\section{2. Éléments de diagnostic et principales leçons}

Des deux côtés de la frontière, les espaces concernés par l'étude partagent un éloignement de leur capitale nationale ainsi qu'un relatif éloignement des nœuds commerciaux et des bassins d'emploi stratégiques. Depuis l'époque coloniale jusqu'à aujourd'hui, ne pouvant que partiellement profiter des flux de marchandises les traversant, ils sont principalement dépendants d'une agriculture pluviale, faiblement mécanisée, fonctionnant surtout sur des filières alimentaires locales et dont les déploiements à l'export sur les marchés internationaux restent limités. Dans ce paysage, la transhumance conserve une place désormais contrainte par une occupation du territoire exerçant une pression sur les pâturages et sur les mouvements des animaux, mais aussi par les limites du dialogue entre les politiques publiques nationales et les difficultés de mise en œuvre de la politique d'intégration régionale.

\subsection{LE POIDS DE LA CROISSANCE DÉMOGRAPHIQUE SUR LES DYNAMIQUES TERRITORIALES}

Les dynamiques territoriales sont structurées depuis 50 ans par le déplacement des zones de culture de l'ouest vers l'est, ainsi que leur densification partout sur le territoire, sous l'effet d'une forte pression démographique. Au nord de la frontière, l'étalement dans le Koulpélogo s'est d'abord réalisé dans la province même, puis de l'autre côté de la frontière, autour de Dapaong. À l'ouest, des réserves naturelles existaient jusque dans les années 2000, mais la savane et les forêts ont peu à peu fait place aux cultures. En 2013, les terroirs semblent saturés dans toute la partie ouest du territoire. À l'est, les grandes plaines relativement bien arrosées mais enclavées et dépourvues d'infrastructure, autrefois clairement propices à la transhumance, sont bordées par des réserves naturelles de faune et de flore dans les deux pays. Pourtant, si la réserve de pâture au nord de la Kompienga demeure préservée, les cultures arrivent aujourd'hui au bord des forêts classées.

Ce processus d'occupation de l'ouest vers l'est devrait perdurer dans l'avenir. Entre 2020 et 2035, la population du territoire pourrait en effet passer de 1,65 million à 2,58 millions d'habitants si les déplacements, et notamment ceux liés à la situation sécuritaire, ne modifient pas les équilibres en place. L'augmentation de la population va se répercuter à la fois en ville et en zone rurale. Les deux populations vont continuer à augmenter fortement, induisant des pressions soutenues sur les ressources naturelles pour l'agriculture et l'élevage dans les campagnes. Sans gain de productivité, la baisse modérée d'agriculteurs dans la population active (en \%) dans les dernières décennies, conduit à devoir «trouver» 350000 ha supplémentaires dans les 15 ans à venir, et 520000 dans les 20 ans à venir. Cela représente, d'ici 2035, la mise en culture de près de 24000 ha supplémentaires par an, contre seulement 14800 ha supplémentaires par an entre 2001 et 2015. 


\section{Carte 3}

Évolution des surfaces des zones de culture 1975-2013
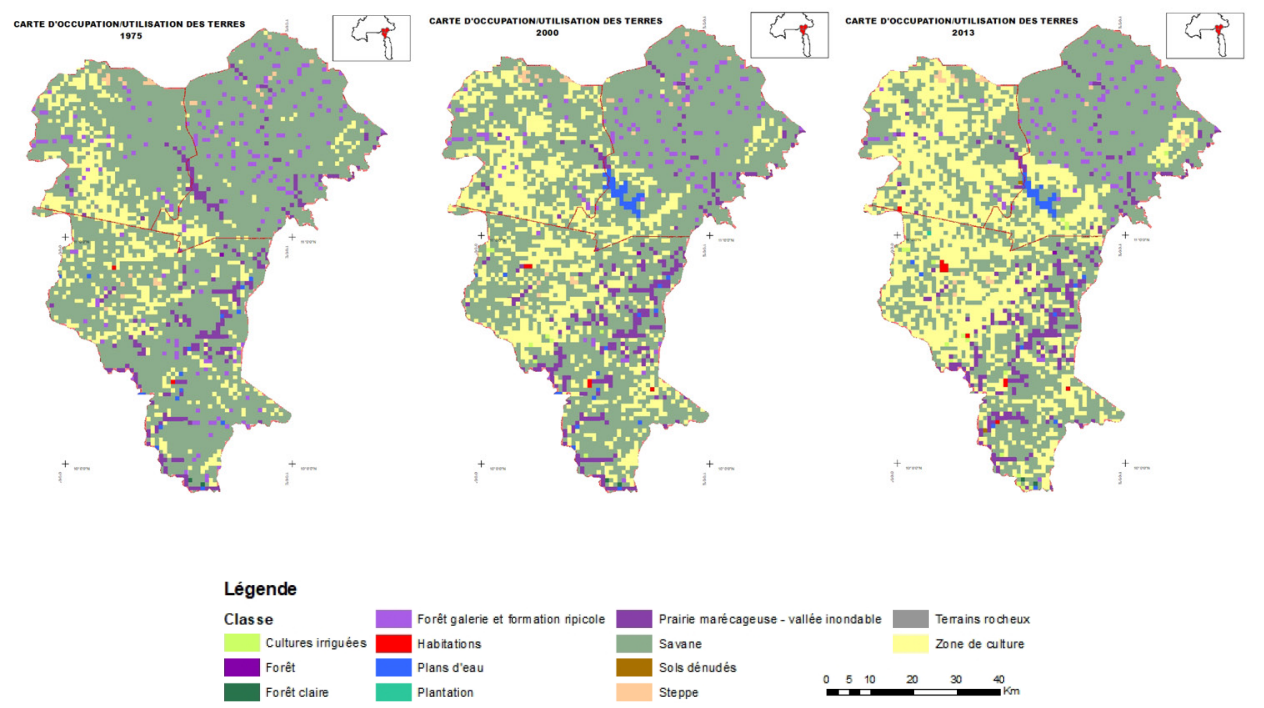

Source: Centre de données EROS du Service géologique des États-Unis (USGS Eros), adaptée par Ibra Touré.

En lien avec la densification massive du territoire, santé, éducation et emploi sont des défis considérables si les prévisions démographiques, et notamment le rajeunissement de la population sur l'ensemble du territoire, se réalisent. Quand 33500 jeunes entraient sur le marché du travail en 2015, ils seront 53400 en 2035, et plus de 60000 en 2040, soit un nombre total d'emplois à créer de près de 750000 d'ici 2035, et 950000 d'ici 2040. Les projections des besoins en personnels et infrastructures de santé et d'éducation, au regard des niveaux atteints lors des dernières décennies, rendent peu probable l'alignement des systèmes sur les standards internationaux. Maintenir la couverture de soins et d'enseignement à son niveau actuel semble d'ores et déjà un objectif ambitieux. Le rythme toujours soutenu de croissance oblige à des arbitrages volontaristes pour assurer l'accès à des services de base. Il demande des engagements politiques dont le territoire ne bénéficie pas aujourd'hui. 


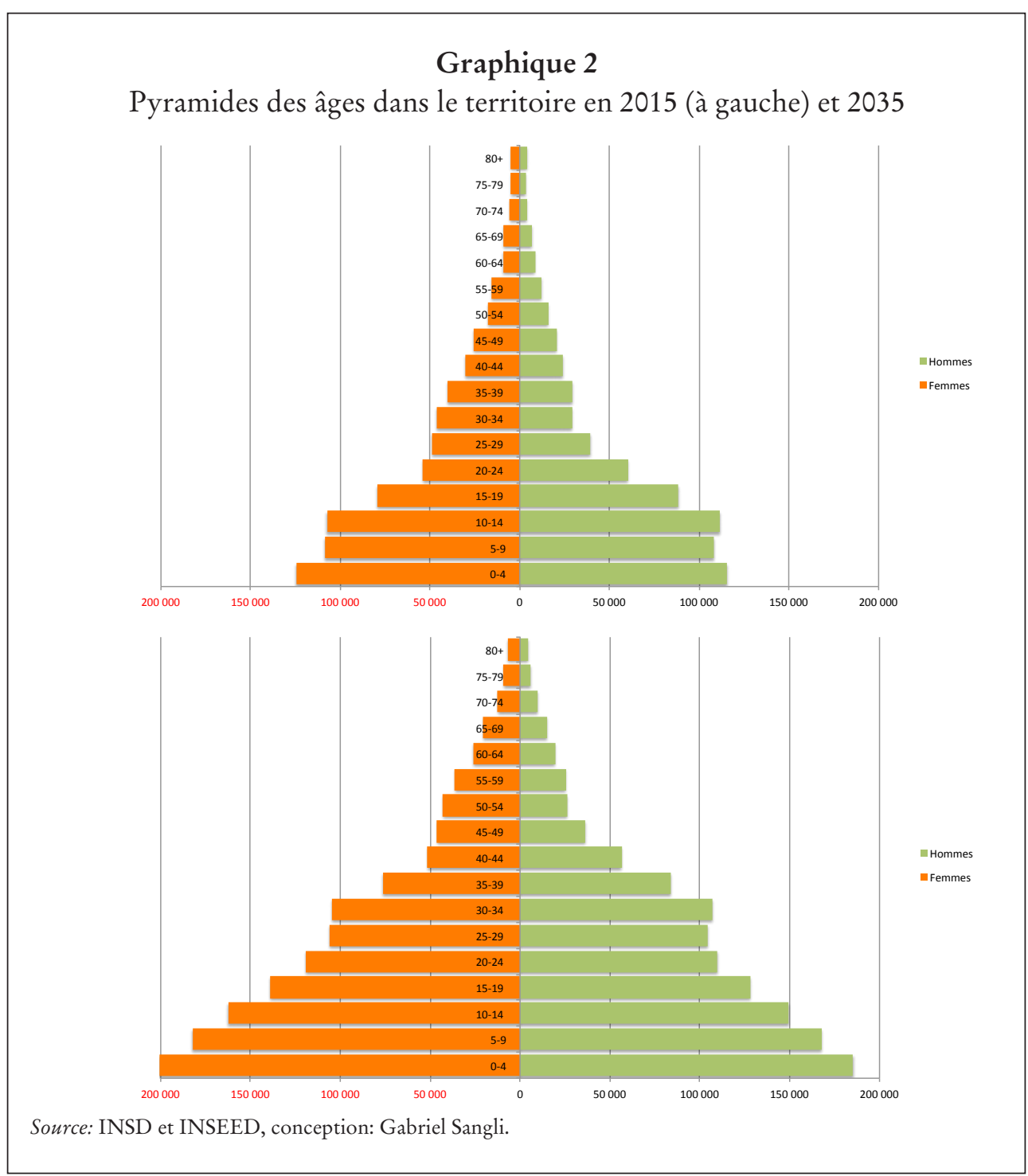

\subsection{LA NÉCESSAIRE MAIS PROBLÉMATIQUE DIVERSIFICATION ÉCONOMIQUE}

L'agriculture et l'élevage occupent près de 90 pour cent des actifs, avec une remarquable permanence. Ils n'enregistrent pas de gains de productivité significatifs; l'accroissement des surfaces suit le croît démographique, avec cependant quelques ajustements dans les systèmes de culture.

On note ainsi le recul sensible du mil, dont les surfaces ont été divisées par 10 en 30 ans, et qui passe de 20 à 5 pour cent des surfaces sur les 20 dernières années.

Cette évolution se fait au bénéfice principal du maïs (avec une multiplication par 4 des surfaces), et à un degré moindre du sorgho et du soja. La réduction de la part du mil est moins marquée au Burkina Faso qu'au Togo, mais la progression du maïs y est tout aussi spectaculaire. Il convient de noter aussi le timide mais réel développement du riz, en particulier dans les Savanes.

Le coton dans les Savanes et le Koulpélogo couvre aujourd'hui près de 20 pour cent des surfaces totales cultivées, même si ses résultats sont instables. L'arachide connaît une régression récente, apparemment corrélée à la progression du sésame. 


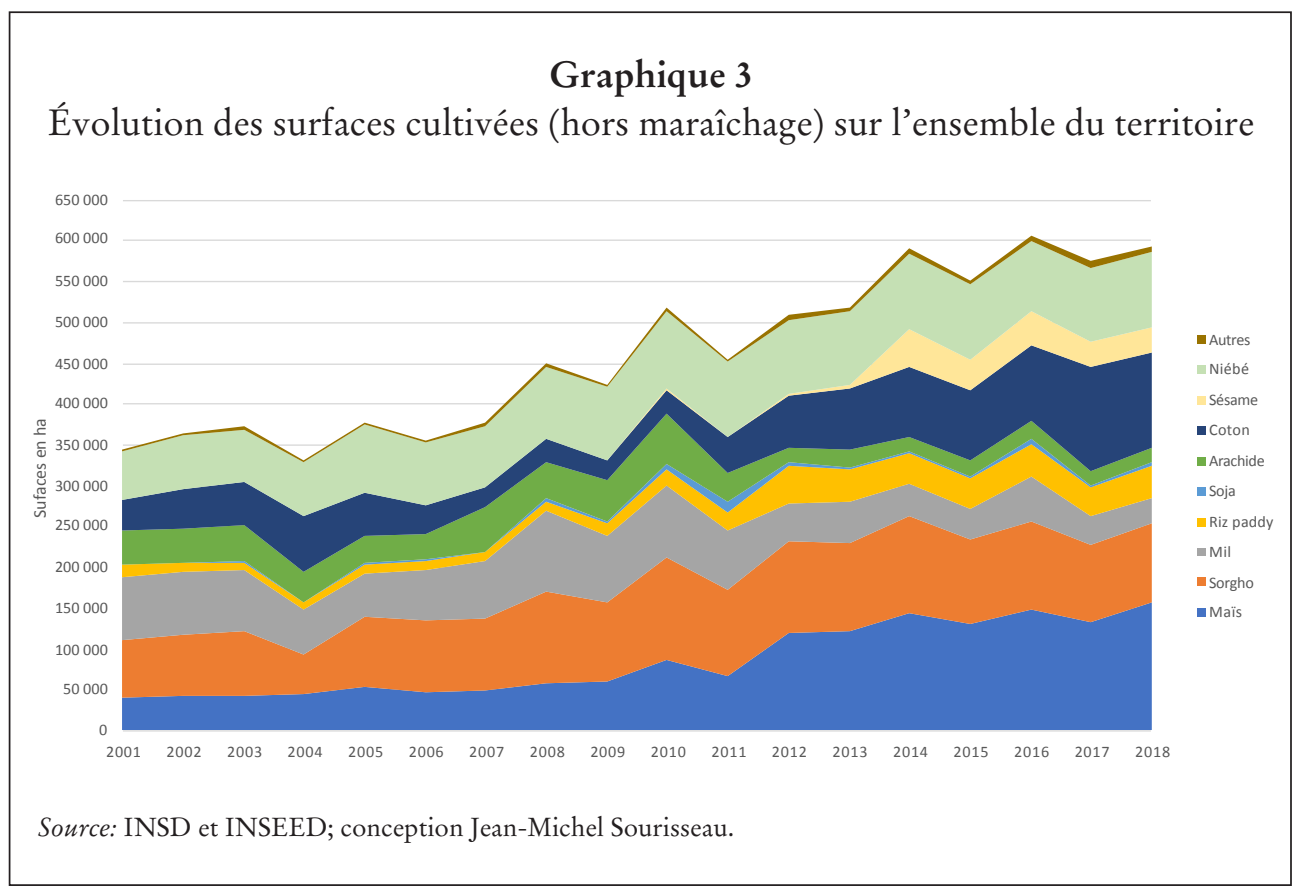

Mais force est de constater que le développement des cultures de rentes à destination des marchés national et international reste en dessous des ambitions affichées; les systèmes restent majoritairement tournés vers l'alimentation des populations du territoire, via les marchés locaux et l'autoconsommation.

Dans l'ensemble des régions du territoire concernées par l'étude, les effectifs du bétail sont en hausse constante. Cette tendance lourde reflète indéniablement une réalité de terrain où le nombre et la taille des troupeaux augmentent. Mais elle reflète aussi une méthode de calcul qui, en l'absence d'un recensement récent, estime à + 2 ou +3 pour cent la hausse annuelle des effectifs. Ces chiffres sont sujets à caution et sont sans doute sous-évalués, à l'instar des données de la zone CEDEAO, elles-aussi régulièrement questionnées.

Pour l'année 2013, pour laquelle nous disposons des données les plus homogènes sur l'ensemble du territoire, la répartition des animaux d'élevage montre la prédominance du Koulpélogo. Les systèmes d'élevage sont dominés par l'élevage agropastoral mobile et allaitant, pour les bovins comme pour les petits ruminants. 


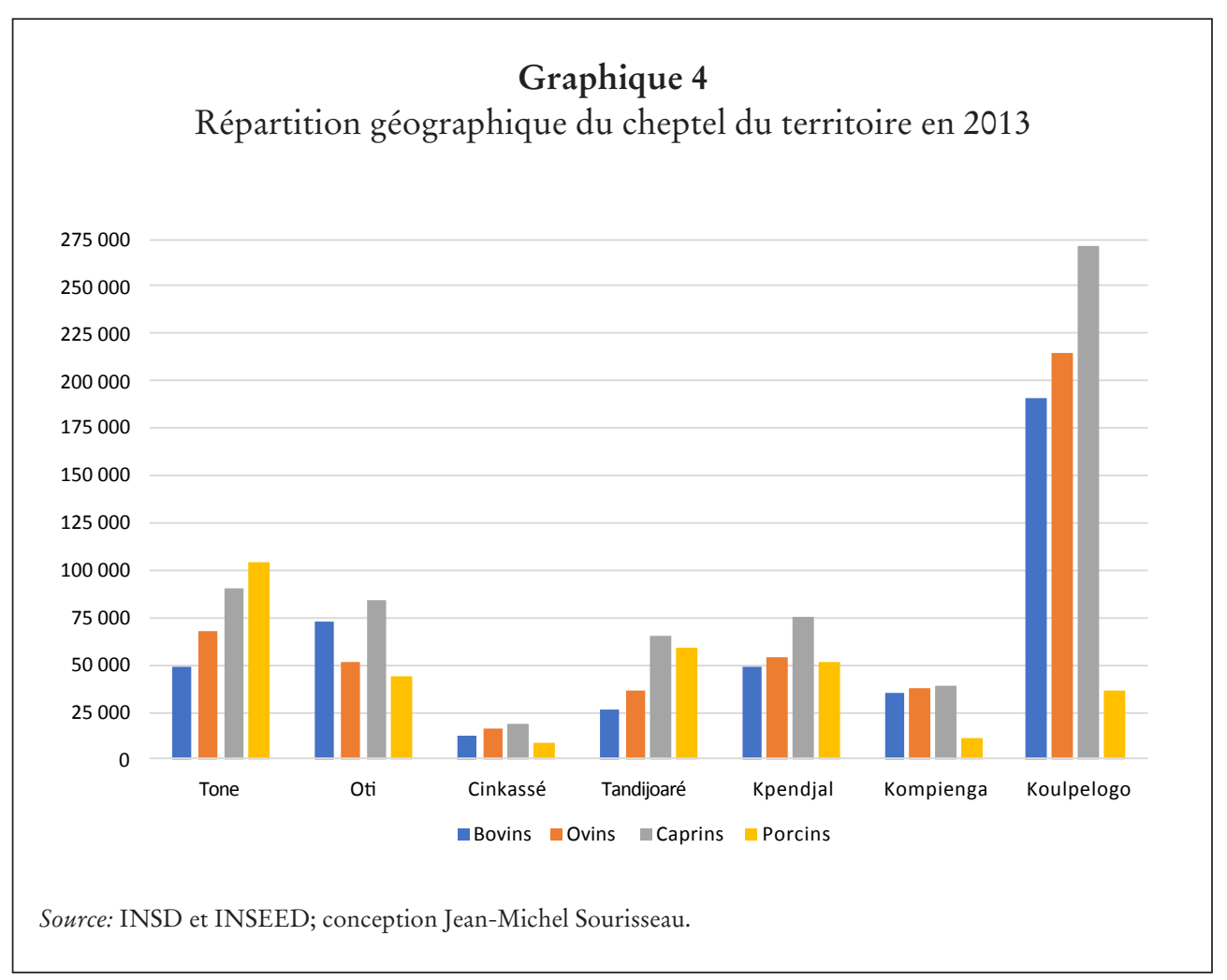

Avec les réserves qui s'imposent sur les valeurs déclaratives, l'élevage est répandu et la répartition du bétail est inégalitaire, surtout au Togo où 5 pour cent des ménages possèderaient 64 pour cent des bovins et 56 pour cent des ménages rencontrés au Togo et 40 pour cent au Burkina Faso élèvent des bovins. Le cheptel moyen par ménage nettement plus important au Togo, où relativement plus de ménages élèvent du bétail, indique un niveau de capitalisation plus élevé puisque souvent ces animaux constituent le capital le plus important de l'exploitation agricole et un capital plus ou moins circulant en fonction des types d'animaux.

Chez les agriculteurs, la présence de bœufs de labour sédentaires, notamment pour la culture du coton, est fréquente et ces animaux sont issus d'un élevage pastoral mobile. L'embouche bovine est rare; l'embouche ovine, plus fréquente, concerne des animaux qui sont engraissés quelques mois à l'auge (sédentarisés). La quasi-totalité de ces animaux ont d'abord été élevés dans un système pastoral transhumant. Les troupeaux ont tous une vocation de production de viande. Quelques animaux sont traits pour une autoconsommation du lait ou une vente de fromage wagashi, surtout dans la région des Savanes. 


\section{Carte 4}

Principales routes et localisation de quelques infrastructures pastorales dans le territoire

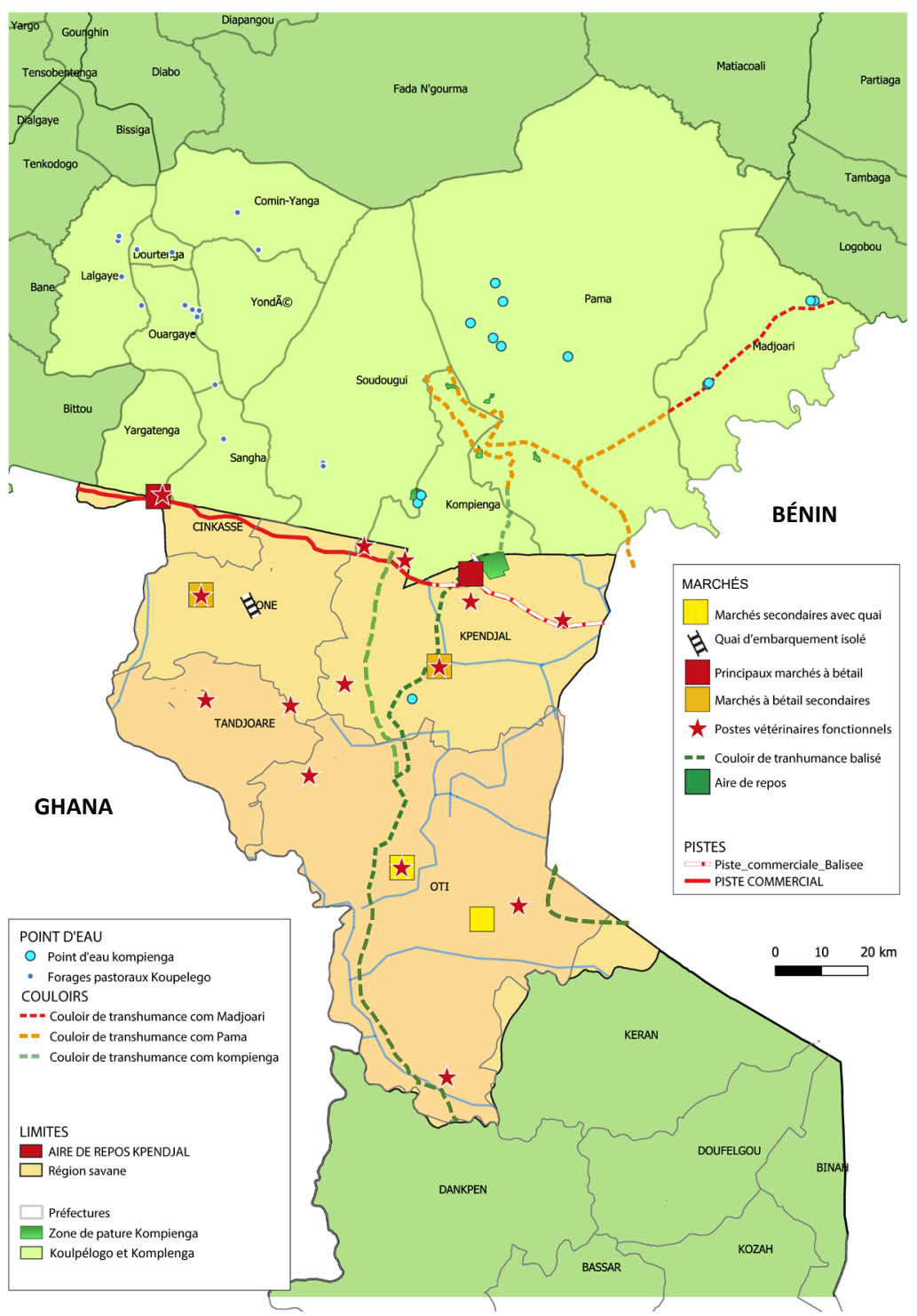

Source: GEVAPAF, conception Jean-Claude Lorente.

Mais les dynamiques de la transhumance modulent cette vision: les informations confirment la permanence de l'élevage mobile et la concentration des mobilités dans l'est du territoire, depuis la Kompienga vers Kpendjal, croisées avec les pistes commerciales d'est en ouest pour rejoindre le marché de Cinkassé.

Les animaux d'élevage concentrés dans le Koulpélogo alimentent un commerce de bétail qui s'intensifie le long de la frontière, en empruntant les pistes du côté du Togo, puis par camion vers d'autres marchés. L'insécurité limite les déplacements nord-sud mais une zone de passage telle que la Kompienga, subissant de plein fouet l'insécurité, demeure centrale dans les transhumances, avec ses infrastructures dédiées. La concentration humaine à Tone et Cinkassé est clairement perçue comme un facteur structurant des marchés des animaux. 
La faiblesse de la diversification économique reste bel et bien une caractéristique forte du territoire, et le signe de marges de manœuvre limitées dans les options stratégiques offertes. Le coton mis à part, mais dont les activités aval ne sont pas localisées sur le territoire, l'agroalimentaire ne trouve pas de filières dans lesquelles se développer. Conjointement, le tissu des entreprises du territoire est peu dense et concerne essentiellement le petit commerce et l'artisanat. À titre d'exemple, la région des Savanes héberge 5,4 pour cent des entreprises du Togo, alors qu'elle représente 12 pour cent de la population du pays. Cette répartition est extrêmement déséquilibrée dans la région, Cinkassé et Tone se partageant près de 90 pour cent des effectifs; 86 pour cent de ces entreprises sont informelles et 99 pour cent sont des petites entreprises unipersonnelles.

La mine ouvre des perspectives potentielles, mais présente aussi des risques indéniables de mauvaise gouvernance; outre les conditions de travail inégales, le secteur attire et favorise depuis quelques années des groupes armés. La fièvre de l'orpaillage n’a pas épargné la province de Koulpélogo. En 2018, une dizaine de mines artisanales étaient recensées dans la zone ${ }^{3}$. La mine industrielle de Youga, sur la frontière avec le Ghana, constitue un pôle d'emploi depuis plusieurs années. Un grand projet minier industriel, porté par le canadien Semafo, déjà propriétaire et exploitant de plusieurs sites, doit par ailleurs voir le jour à Nabanga, dans la province de la Kompienga.

Pour autant, et malgré cette activité, la part de la population active trouvant de l'emploi dans le secteur reste faible. Estimée à moins de 1 pour cent en 2005 pour l'ensemble de la région du Centre-Est, elle a pu augmenter ces dernières années avec le boom qu'a connu le secteur, mais elle reste évaluée à moins de 5 pour cent.

De même, les promesses d'un tourisme organisé autour des parcs nationaux dans la zone toute proche sont pour l'heure non tenues, du fait d'une pression humaine peu maitrisée, mais surtout parce que ces zones au Burkina Faso sont aujourd'hui des bases arrière des terroristes.

\subsection{APERÇU SUR LES CONDITIONS DE VIE: DES TERRITOIRES MARGINALISÉS DANS LEUR ESPACE NATIONAL}

La région des Savanes est depuis longtemps bien plus «pauvre» que le reste du Togo, et bien plus inégalitaire que les régions de l'Est et plus encore du Centre-Est du Burkina. Pour autant, les chiffres disponibles récents semblent attester d'un rattrapage national sur cet indicateur, ainsi que d'une homogénéisation des taux entre les trois régions.

Les différentes enquêtes nationales auprès des ménages insistent en particulier sur la trajectoire positive de la région Est au Burkina, tirée par le développement de Fada. S'il est difficile de juger de l'impact de la crise politique de 2015 et de la dégradation plus récente de la situation sécuritaire sur les revenus et la pauvreté des ménages du territoire, il est à craindre que le ralentissement économique objectivement observé ait déjà eu des répercussions sur les indicateurs de bien-être. Il est à craindre aussi que les écarts entre le Burkina Faso et le Togo se soient creusés en termes de revenus et d'inégalités.

https://www.lepoint.fr/economie/burkina-faso-boom-minier-la-malediction-n-est-pas-loin-14-09-2018-2251158_28.php consulté le 1er décembre 2019. 


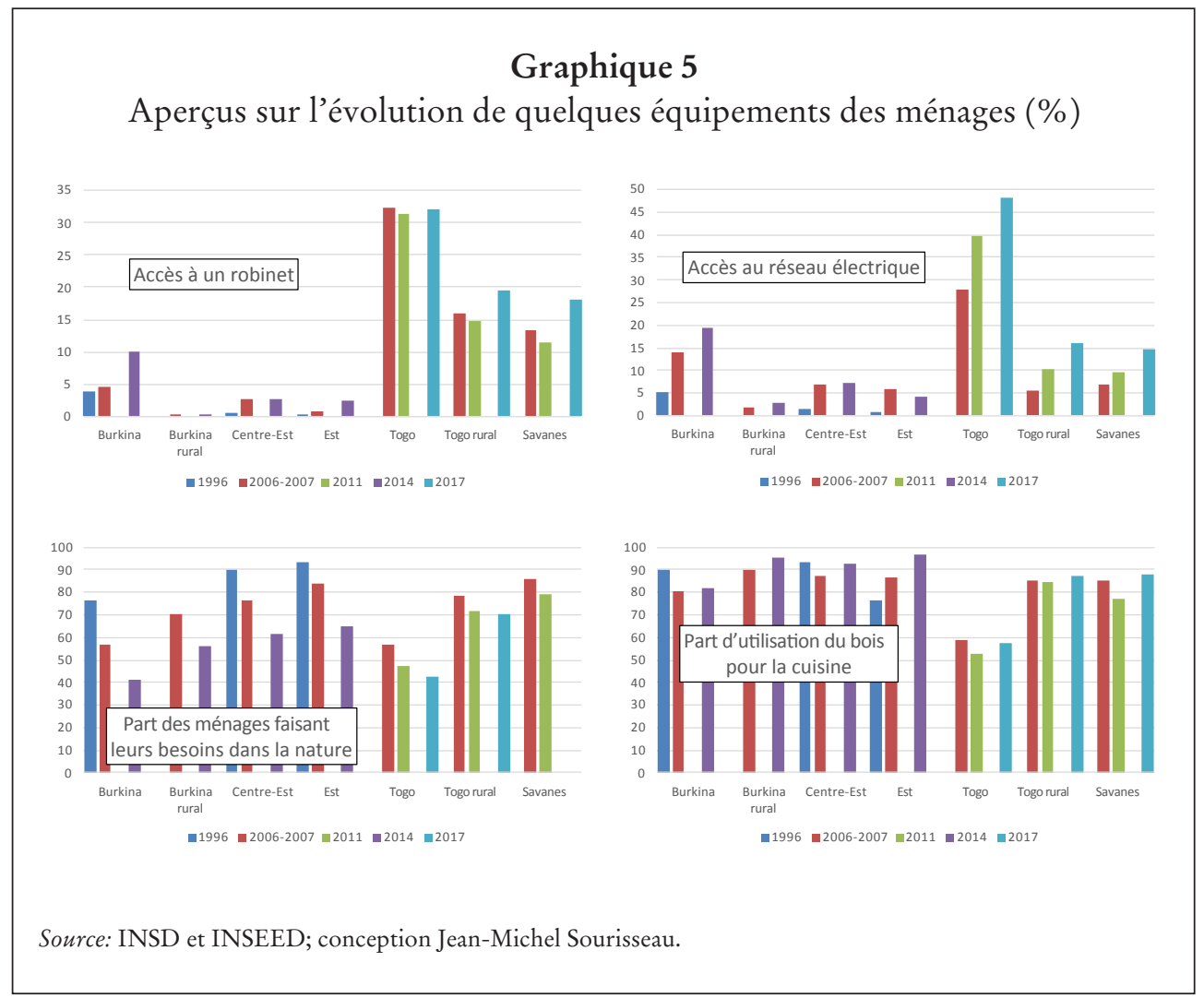

Les résultats sur l'équipement des ménages insistent sur le retard considérable, sur l'ensemble des indicateurs, de notre territoire par rapport aux données nationales. Au Burkina, les régions Est et Centre-Est ont en revanche des équipements globalement de meilleure qualité que la moyenne du monde rural, alors que les Savanes sont plutôt en retard par rapport au Togo rural.

\subsection{DES ENQUÊTES TÉMOIGNANT DE REPRÉSENTATIONS CONTRASTÉES SUR L'AVENIR DU TERRITOIRE}

\section{Les représentations d'un échantillon de ménages ruraux}

Les enquêtes effectuées auprès de l'échantillon de ménages ruraux dans le territoire confirment les conditions de vie difficiles, mais ont permis aussi de mieux comprendre comment les individus se représentent la trajectoire de leur territoire d'appartenance, ainsi que ses avenirs possibles.

Les hommes chefs de famille enquêtés ont une perception de la taille idéale de la famille avec en moyenne deux épouses et environ 5,5 enfants par épouse, soit des familles de 13 personnes en moyenne. Selon toutes les personnes interrogées, le nombre d'enfants par femme serait en diminution durant la dernière décennie. Les jeunes de notre échantillon estiment le nombre idéal à 4,5, mais il reste encore de 6,5 pour les femmes interrogées. Le planning familial reste encore méconnu pour une partie de la population, y compris chez les jeunes, tandis que seules 24 pour cent des femmes déclarent avoir déjà consulté. Ces perceptions, avec toutes les limites liées à l'enquête, indiquent que la transition démographique devrait être lente et progressive. 
Les parents interrogés sur l'avenir de leurs enfants anticipent un exode rural très important, essentiellement vers la capitale mais aussi vers les villes secondaires (en particulier au Togo). Et de fait, confirmant la vision de leurs parents, seulement 24 pour cent des jeunes interrogés envisagent de rester vivre au village; ils déclarent vouloir vivre en ville à 81 pour cent au Togo et à 72 pour cent au Burkina Faso. Mais pour l'instant, dans les familles interrogées, les jeunes partis en ville sont beaucoup moins nombreux que ce que les parents anticipent pour les plus jeunes enfants encore à charge. En effet, 55 pour cent des personnes au Togo et 44 pour cent au Burkina Faso sont restées dans la même préfecture ou la même commune (alors que pour les parents les réponses donnaient respectivement 20 pour cent et 5 pour cent); 28 pour cent sont partis à l'étranger, signe d'une mobilité transfrontalière de proximité très active.

Les personnes enquêtées jugent en général les infrastructures économiques absentes ou en mauvais état et, dans tous les cas, insuffisantes. Au Togo, l'accès à l'eau et à l'électricité est jugé particulièrement problématique, quant au Burkina l'insatisfaction porte surtout sur les voies de communication.

Peu d'exploitations agricoles enquêtées pratiquent la transhumance, mais il est intéressant de noter que la pratique concerne davantage de ménages au Togo (13 pour cent de notre échantillon) qu'au Burkina Faso, où les ménages estiment la mobilité animale en léger déclin. Intéressante aussi est l'importance des relations déclarées avec les transhumants, en particulier sous forme de relations commerciales avec la vente ou l'achat de produits agricoles. En contre-point, l'enquête révèle à la fois l'exacerbation des conflits autour de la transhumance, mais aussi une très grande méconnaissance du secteur des personnes jugeant les conflits en augmentation. L'immense majorité des conflits se règlerait à l'amiable au Togo (plus de la moitié au Burkina), avec des fréquences d'occurrence très variables. Au final, les ressentis des ménages interrogés laissent à penser qu'un dialogue apaisé est possible à travers une meilleure connaissance de la réalité de la transhumance et une plus grande médiatisation des instruments de résolution des conflits déjà existants, et notamment les cadres de médiations ouverts par les collectivités locales à l'initiative des comités nationaux de transhumance.

Parmi les évènements qui ont marqué le territoire dans le passé selon les personnes enquêtées, on note l'importance des évènements qui ont directement frappé la famille avec en premier lieu les évènements de type démographique (décès, maladies, accidents), avec parfois des effets cités qui concernent un appauvrissement ou une dislocation de la famille. Les évènements climatiques sont cités essentiellement au Burkina Faso comme si les ménages du Togo avaient été moins impactés ou sensibles à ce type d'évènements.

Mais paradoxalement, les ménages togolais sont plus inquiets que les Burkinabè quant aux impacts à venir du changement climatique et de l'insécurité, alors que les Burkinabè semblent surtout sensibles au manque de perspective économique. On note aussi que la plupart des évènements cités sont récents ou même très récents traduisant, peut-être, une perception du territoire basée sur le présent et pas du territoire comme le résultat d'une construction progressive marquée par une succession d'évènements sur le temps long. 


\section{Les représentations des personnes ressources interrogées}

L'enquête qualitative auprès de 61 experts du territoire témoigne d'une intégration très largement inachevée entre les entités administratives qui composent l'espace transfrontalier. Même entre les provinces burkinabè, l'appartenance à des régions différentes génère des connaissances et des collaborations seulement partielles. La plupart des personnes interrogées, même celles s'exprimant sur l'ensemble de la zone, restent concentrées sur leur espace immédiat de proximité.

Cette intégration inachevée contraste pourtant avec la grande mobilité de part et d'autre des limites administratives, tant des biens que des personnes. Toutes les marchandises, productions agricoles comme biens manufacturés, sont concernées. Certes, ces échanges marchands sont partiellement illicites, mais les entretiens insistent sur le dynamisme et les importants flux de marchandises transitant par Cinkassé ou dans une moindre mesure par Sanga et Pogno. De même, les personnes sont extrêmement mobiles, notamment pour la recherche d'emploi. Bien entendu, ces mobilités et échanges s'inscrivent dans un cadre plus large. Ainsi le départ de jeunes de la zone vers le Ghana et la Côte d'Ivoire pour trouver des emplois semble un processus de plus en plus structurant, et les mobilités vers le Bénin, et en particulier aujourd'hui via Mandouri pour le Togo, s'intensifient. Il n'en reste pas moins que les entretiens traduisent l'existence d'une réelle identité territoriale, fondée d'une part sur l'éloignement des pouvoirs centraux, mais aussi la circulation et les échanges économiques et sociaux locaux et transfrontaliers, et qui offre indéniablement des leviers nouveaux et prometteurs pour le développement rural. 


\section{Enseignements des ateliers d'anticipation: aide à la décision et passage à l'action}

La projection participative dans le futur du territoire s'est faite en deux étapes articulées. Dans un premier temps, à partir du diagnostic (brièvement présenté ci-dessus) et des premiers travaux en ateliers, des images plausibles du territoire transfrontalier en 2035, incluant la place, le rôle et les fonctions que la transhumance pourrait avoir dans ces différents scénarios, ont été imaginées et mises en récit. Puis il a été discuté d'actions dans le domaine de la gouvernance locale et de la coopération transfrontalière pour qu'une transhumance apaisée puisse être envisagée.

\subsection{LA CO-ÉLABORATION PARTICIPATIVE DE SCÉNARIOS}

Une succession de dix sessions de travail collectif a permis au groupe d'experts de construire des images contrastées du futur du territoire, aboutissant à l'écriture d'histoires du futur incluant différentes formes de transhumance et leur participation au territoire.

- Session 1. Définition du système et de la question: le diagnostic, restitué au début de l'atelier, a permis de stabiliser la problématique et de préciser l'horizon temporel d'anticipation.

- Session 2. Introduction à la question et à la démarche de l'anticipation: il s'est ici agi de réaliser une fresque historique qui retrace les évènements externes et internes qui ont influencé le territoire, et d'identifier les espoirs et craintes relatifs à des évènements qui pourraient se produire.

- Session 3. L'identification des facteurs de changements du territoire: ces forces correspondent à des dynamiques passées, présentes et futures, perçues comme ayant potentiellement une influence sur l'évolution du territoire.

- Session 4. Identification des forces motrices (analyse structurelle): la classification des forces en différents groupes (motrices, leviers, produits, singulières) permet de caractériser la structure du système et de sélectionner les principales forces dites «motrices»: celles qui ont une grande influence sur les autres et en sont peu dépendantes.

- Session 5. Définition des états du futur (analyse morphologique): pour chaque force motrice sélectionnée, on explore un jeu d'hypothèses contrastées appelées aussi états du futur plausibles.

- Session 6. Co-élaboration des trames de scénarios: une trame est une combinaison intégrant une hypothèse d'évolution pour chacune des forces motrices, combinaison présentée sous forme de synopsis décrivant une situation future de la région transfrontalière Togo-Burkina en 2035.

- Session 7. Écriture des synopsis: les trames sont développées sous forme de synopsis, des paragraphes courts combinant les différents états du futur de chaque force motrice. 
- Session 8. Développement des narratifs: les synopsis sont développés en narratifs complets sous la forme d'un texte cohérent et plausible dans lequel les experts incorporent les autres facteurs de changement.

- Session 9. L'intégration de la variable transhumance: à l'issue du développement de tous les narratifs, le groupe a procédé au choix des scénarios plus contrastés pour réintégrer la question de la transhumance.

- Session 10. Introduction aux approches de back-casting: cette session a permis aux participants de se projeter dans l'utilisation des scénarios pour changer le présent.

Les huit forces motrices, sur lesquelles les différentes images du futur du territoire ont été construites, sont les suivantes:

- Capital humain: qualité de l'éducation et de la formation des populations et capacité des populations à s'impliquer dans le développement du territoire.

- Gouvernance locale: qualité de la gouvernance locale dans le territoire: renouvellement des élus, redevabilité, transparence, participation, etc.

- Sécurité: niveau de sécurité des biens et des personnes et collaboration entre services de sécurité de part et d'autre de la frontière.

- Croît démographique: niveau de croissance naturelle de la population.

- Structuration professionnelle: qualité de la structuration des organisations professionnelles locales (agriculteurs, éleveurs, artisans, commerçants, pêcheurs, exploitants forestiers, etc.).

- Activités minières: niveau de développement de l'activité minière et de la qualité de sa gestion.

- Coopération décentralisée transfrontalière: niveau et qualité de la coopération entre les collectivités territoriales de part et d'autre de la frontière.

- Préservation des écosystèmes: l'état des écosystèmes: couverture végétale, biodiversité faunique, floristique, lacustre.

Quinze scénarios ont été construits en combinant différents états du futur de ces huit forces motrices, puis le groupe d'experts a travaillé plus précisément sur huit de ces scénarios pour y intégrer la transhumance. À titre d'exemple, deux de ces images du territoire en 2035, intégrant les possibles rôles et fonctions possibles de la transhumance, sont présentées ici. Rappelons que les scénarios souhaitables imaginés supposent les problèmes résolus par des politiques vertueuses. Tout l'enjeu des séances qui ont suivi est de formuler des pistes d'incitation pour déverrouiller les contraintes majeures qui ont prévalu jusqu'ici. 
Enseignements des ateliers d'anticipation: aide à la décision et passage à l'action

«Oasis du désert»

En 2035, le territoire transfrontalier entre le Togo et le Burkina est un vaste espace quasi-désertique caractérisé par la faiblesse de ses flore et faune, et le tarissement des plans d'eau. L'activité principale demeure l'élevage avec une forte propension ancestrale vers la transhumance transfrontalière. L'effectif du bétail de la zone est évalué à plus de 850000 UBT. Longtemps handicapé par la rareté des ressources hydriques et alimentaires, l'élevage bénéficie au cours de ces dernières années d'un important investissement permettant une semi-sédentarisation. Une agriculture de subsistance avec des technologies adaptées au Sahel (irrigation, zaï, demi-lune, etc.) est pratiquée. Des techniques adaptées de culture fourragères permettent aux éleveurs de faire du bon foin et de constituer un stock important dans les magasins de la coopérative.

En outre, la coopération transfrontalière est dynamique et performante, caractérisée par des jumelages, des ententes et des intercommunalités entre les collectivités, permettant la planification commune de projets. Dans les nombreux projets de cette coopération, un accent particulier a été accordé aux défis de la mobilité du bétail. Ainsi, des pistes pastorales balisées, des aires de repos, des forages solaires, des parcs de vaccination ont été mis en place facilitant la mobilité du bétail vers les points d'oasis. À la croissance démographique forte s'ajoute un flux migratoire important lié aux activités minières et à la prospérité de la transhumance. Les ressources humaines ont une éducation et formation professionnelle de qualité qui est en adéquation avec les besoins effectifs et pour tous. Cependant, la pratique de la transhumance soustrait une partie des éleveurs $d u$ système éducatif classique, ce qui a conduit à la mise en place de centres d'alphabétisation et de formation professionnelle adaptés aux besoins des éleveurs.

Les ressources minières sont exploitées par des procédés modernes respectant le développement durable avec une gestion transparente permettant à toute la population de bénéficier des retombées positives. La décentralisation est une réalité et les principes de redevabilité, de transparence, de participation, d'équité genre et d'opérationnalité sont respectés, la gouvernance est donc assurée par les élus locaux. Ces élus locaux, avec l'appui des partenaires techniques et financiers et du gouvernement, ont, au cours de leurs mandats, permis la construction et la fonctionnalité de diverses infrastructures socioéconomiques. Parmi ces infrastructures on note: des marchés à bétail transfrontaliers où les produits d'élevage se vendent et s'exportent facilement et sans tracasserie vers les zones côtières; des infrastructures de stockage de céréales, d'aliments bétail, de médicaments vétérinaires, autogérées par des centres d'autopromotion féminine et des auxiliaires villageois d'élevage; des centres de distribution d'énergie renouvelable et d'eau potable pour toute la population; des postes vétérinaires bien équipés et dotés de personnels qualifiés.

Pour donner une impulsion particulière au secteur de l'élevage et du pastoralisme, les infrastructures pastorales mises en place dans le cadre de la coopération transfrontalière (pistes pastorales, parc, forage) sont gérées par les élus locaux et les associations professionnelles d'éleveurs. Toutes ces infrastructures permettent aux éleveurs de conduire, dans le même territoire et sans contrainte majeure, leur troupeau d'une zone à l'autre et autour des points d'oasis où se développe la culture fourragère. Des marchés de bétail connaissent une grande affluence et les agriculteurs en profitent pour acheter des taurillons pour la culture attelée. En contrepartie de ce lourd investissement en faveur du pastoralisme, les communes bénéficient de plusieurs retombées positives de cette mobilité à travers des taxes de mobilité et des taxes liées aux ventes dans les marchés de bétail. Ces taxes ensemble constituent près de 60 pour cent du budget de la commune.

Les organisations professionnelles locales sont bien organisées et dynamiques. En partenariat avec les forces de sécurité, les OP ont initié un système permettant le déplacement sécurisé des transhumants. Ce système repose sur l'utilisation obligatoire de badge d'identification. L'apport diversifié de la transhumance sur les différents maillons de l'économie (agriculture, commerce, fiscalité,) a contribué à une réduction de la pauvreté sur le territoire qui se situe autour de 3 pour cent. Une stratégie de défense transfrontalière dans laquelle les communautés collaborent avec les forces de sécurité et incluant l'utilisation de robots permet une absence de conflits communautaire et la libre circulation des personnes et des biens dans un climat sécurisé. Cette stratégie mise en place éloigne le territoire de l'attaque des terroristes que connaissent certains territoires de la sous-région. 
«La face du chaos»

\section{"Quand I'homme contredit la générosité de la nature, le chaos est presque toujours à l'affût.»}

En 2035, la région transfrontalière entre le Togo et le Burkina est caractérisée par un écosystème (forêts, animaux, cours d'eau) luxuriant avec des ressources étendues et diversifiées qui fonctionnent de manière équilibrée. La zone compte non seulement des parcs et des réserves mais aussi d'immenses espaces pastoraux et cultivables. Ce potentiel naturel attire des éleveurs transhumants de toute la région avec les avantages socioéconomiques afférents. Ainsi, les relations entre les transhumants et les populations permettent à ces derniers de bénéficier des effets de l'élevage dans l'agriculture et aussi des échanges commerciaux entre ces deux communautés.

Mais des menaces pèsent sur cet atout de la zone. En effet, il n'existe pas de coopération intercommunale car les communes sont reversées dans l'administration centrale. II n'y a aucune vision stratégique commune de développement à l'échelle du terroir, autant dans le domaine des échanges commerciaux que dans celui du développement des filières du territoire et des infrastructures agro-sylvo-pastorales et halieutiques. Ainsi, la transhumance est mise à mal par ce manque d'organisation du terroir. Les éleveurs sont persécutés dans la zone et certains préfèrent s'éloigner de ce terroir, fuyant les multiples taxes et contraintes liées à leur libre circulation dans le terroir.

La population est dans une situation d'insécurité généralisée à cause de la rupture de collaboration transfrontalière entre les services de sécurité et la population, et des tensions au sein des services de sécurité. Ceci met à mal la cohésion sociale et l'intégration entre les peuples de part et d'autre de la frontière. Ce manque de collaboration est d'autant plus contraignant pour les transhumants obligés de se déplacer de part et d'autre de la frontière à la recherche de pâturage. Ces derniers sont même accusés, à tort ou à raison, de braquage, vol, kidnapping et autres activités illicites comme la divagation des animaux dans les champs, l'abattage d'arbres pour le fourrage, etc.
Le territoire est dominé par des potentats locaux. La gestion des biens communs est familiale et ethnocentrique avec une confiscation des ressources par des pseudos propriétaires terriens organisés et violents, ce qui empêche l'exploitation des richesses minières. Difficile de trouver des moyens pour introduire des technologies nouvelles dans les domaines agricoles et l'élevage, secteurs les plus importants dans le terroir. Plus grave, les populations sont privées d'infrastructures et équipements de services sociaux de base.

Les organisations professionnelles sont disloquées et la représentation des acteurs dépend des individus ou d'organisations familiales. Les filières sont dominées par les acteurs privés et politiques. Les producteurs ne bénéficient d'aucun accompagnement, pas même d'infrastructures de stockage de leurs produits, ni d'assistance médicale pour le bétail. Tout ceci rend non seulement difficile l'activité de transhumance dans la zone, mais elle ne profite qu'à quelques individus qui négocient individuellement avec les transhumants et en tirent profit au détriment de la communauté.

Pour ne rien arranger, on assiste à une forte croissance démographique, un indice synthétique de fécondité élevé dans un contexte de rareté de services sociaux. Une situation qui aggrave la difficulté d'accès à l'emploi, d'autant plus que rien n'est entrepris pour accompagner l'industrialisation.

Dans un autre registre et non des moindres, on note une instabilité politique à l'échelle du territoire, des menaces sur la cohésion sociale, l'ignorance de concept comme l'inclusion genre. La présence des transhumants, loin d'être un atout, est perçue comme un sérieux problème dans le terroir. Rien d'étonnant, les populations sont formées dans des systèmes éducatifs obscurantistes... La face du chaos. 


\subsection{APERÇU DE QUELQUES RECOMMANDATIONS POUR L'ACTION}

L'atelier dédié aux recommandations pour l'action s'est attaché à valoriser la dynamique de groupe construite durant les deux premières semaines de travail collectif, autour de trois sessions de travail:

- Session 11. Choix du périmètre des recommandations et définition d'une vision. Les histoires du futur peuvent être utilisées pour éclairer les décisions des acteurs, simplement par mise en circulation et débats publics. Mais les histoires peuvent aussi apparaitre comme des visions à atteindre et inspirer des politiques publiques à mettre en œuvre. Dans cette perspective, nous avons sélectionné une histoire inspirante parmi les huit - «Oasis du désert» - et précisé dans ce futur, une vision à atteindre: «En 2035, une gouvernance territoriale autonome, démocratique et compétente est mise en oeuvre dans la région transfrontalière du Togo et du Burkina Faso. Des cadres de concertations intégrés et multisectoriels sont effectifs. La sécurité est assurée et la transhumance est apaisée.»

- Session 12. Les conditions de l'action (acteurs, changements, contraintes et opportunités). Les acteurs les plus importants pour réaliser la vision ont été identifiés, et les changements à opérer et les obstacles à éviter précisément définis.

- Session 13. Les stratégies et les recommandations pour l'action. Durant cette dernière session, les «experts» se sont concentrés sur l'écriture de propositions très concrètes d'actions, pour améliorer la gouvernance transfrontalière dans les domaines de la sécurité, de la coopération entre les collectivités locales et de la transhumance.

Les actions préconisées concernent cinq grands domaines, reconstitués après que les experts se soient exprimés sur des propositions plus spécifiques: la production de textes réglementaires transfrontaliers; la formation et la sensibilisation à la bonne gouvernance; la production institutionnelle et les mécanismes de suivi; le financement de la gouvernance et de la coopération; la production et la gestion des données et des connaissances pour la gouvernance et la coopération.

Ces domaines renvoient à des politiques publiques classiquement convoquées pour le développement rural et l'intégration régionale, mais qui n'ont pas, selon les experts, pu être efficacement investis par le passé. Leur formulation réaffirme l'ancrage du groupe à un cadre administratif dont les fondements et la légitimité ne sont pas questionnés. Pour autant, de nombreuses recommandations proposent une nouvelle production institutionnelle, plus efficace car partant de la valorisation d'atouts spécifiques d'un territoire jusqu'alors peu pensé et intégré dans la fabrique de l'action publique.

À titre d'exemple, le schéma ci-après reprend les recommandations formulées pour le domaine de la production institutionnelle et les mécanismes de suivi.

Les actions proposées ne couvrent qu'une partie des champs possibles d'intervention (la gouvernance locale et la coopération transfrontalière) et sont encore à travailler, y compris pour trouver des voies de portage. Pour autant, elles constituent une base solide pour envisager l'avenir. 


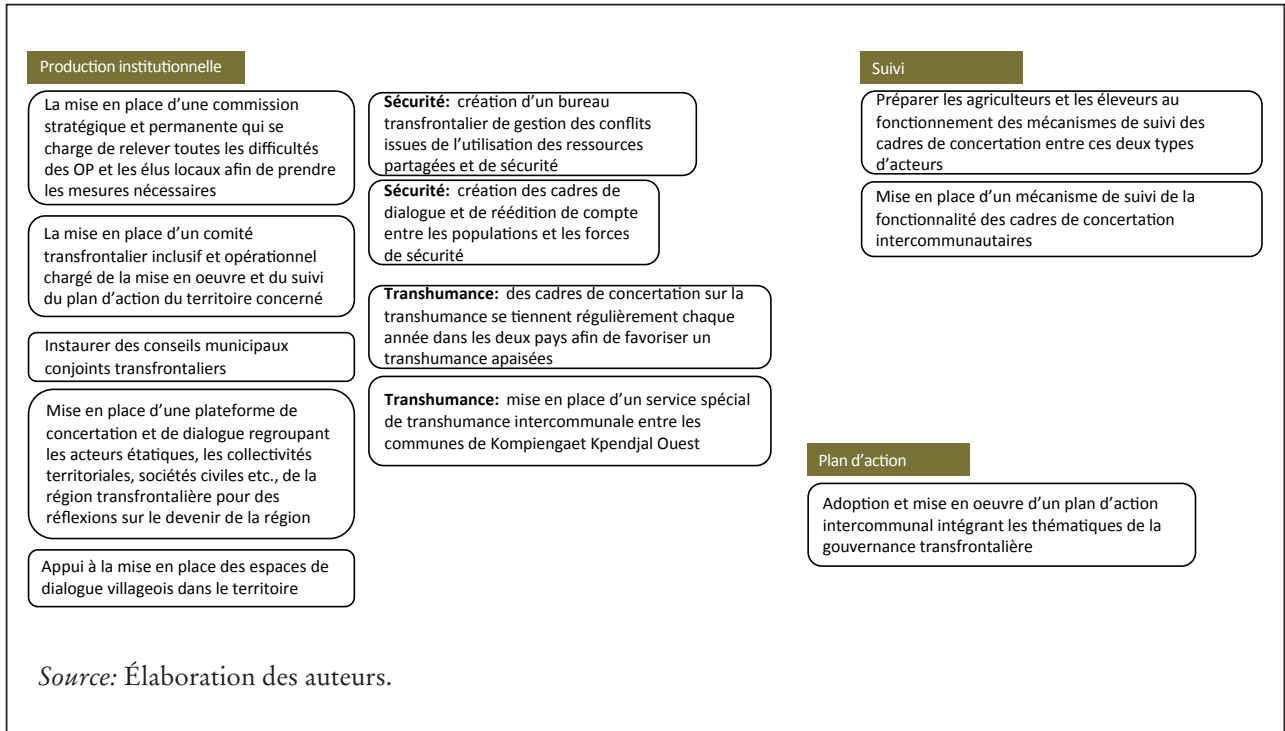

\subsection{LES PERSPECTIVES OFFERTES POUR LE TERRITOIRE PAR LE TRAVAIL PARTICIPATIF RÉALISÉ}

Les scénarios construits ne sont pas des prédictions et il est peu important qu'ils se produisent ou non tels qu'ils ont été formulés. De même, les recommandations formulées demeurent incomplètes et partielles, ne touchant que les questions de la gouvernance locale et de la coopération transfrontalière.

Ce qui importe, c'est la connaissance collective progressivement construite qui permet au groupe d'experts, de profils extrêmement divers et réunis pour l'occasion, d'avoir de nouvelles représentations de leur territoire, de la transhumance dans ce territoire, des grandes dynamiques à l'œuvre et des processus pouvant changer les trajectoires aujourd'hui à l'œuvre. Ils disposent de nouveaux éléments pour agir dans le présent, pour orienter le futur dans des directions souhaitées, ou au moins pour éviter un glissement vers des directions non désirables.

Surtout, sur la base d'une information à jour, partagée et construite collectivement sur le territoire (enquêtes et diagnostic), complétée par l'expression collective de positions sur des enjeux définis ensemble (ateliers), le travail conduit ouvre des perspectives pour que les experts impliqués dans cette démarche, ensemble ou non, entre eux ou en associant d'autres réseaux d'appartenance, puissent se servir du futur pour transformer le présent. Il semble à l'équipe de facilitation qu'une dynamique collective est née, qu'un cadre d'action transfrontalier inédit est en gestation, qui laisse envisager de nouvelles modalités d'action et surtout de collaboration de part et d'autre de la frontière. Le groupe d'experts a pu aborder, non sans tensions mais avec écoute réciproque et compréhension, des sujets extrêmement conflictuels. En se projetant dans le futur et en repositionnant la transhumance dans ses rôles et fonctions pour l'ensemble du territoire transfrontalier, un dialogue s'est engagé entre élus des deux pays, entre agriculteurs et pasteurs, mais aussi commerçants, enseignants, services déconcentrés de l'État, etc.

L'impact réel de cette construction sur le futur du pastoralisme dépendra bien entendu de dynamiques extérieures au territoire, sur lesquelles les acteurs locaux ont peu ou pas de poids (les forces externes dans le vocabulaire de la prospective). Il convient en particulier de rappeler que ce territoire est enchâssé dans un sous-espace 
régional qui influe sur sa trajectoire. Mais ces forces externes ne doivent pas occulter les réelles marges de manœuvre locales, qui reposent sur la capacité et la volonté de ces mêmes acteurs, relayés ensuite par d'autres acteurs du développement, à faire vivre la dynamique de travail et de réflexion entamée lors des ateliers. La prospective territoriale est susceptible d'initier un processus de changement; il appartient aux personnes directement et indirectement impliquées dans le territoire de le concrétiser, dans les espaces politiques qu'ils maitrisent. 



\section{Quelques perspectives pour le territoire et le pastoralisme}

\subsection{RECONNAÎTRE ET VALORISER LES RESSOURCES TERRITORIALES PAR DES STRATÉGIES RÉSOLUMENT TRANSFRONTALIÈRES}

Le territoire gagnerait à viser une diversification économique s'appuyant sur ses ressources spécifiques pour sortir de la dépendance au commerce transfrontalier ou plutôt à ses retombées fragiles - comme principale source de revenus. Ce sont bien les femmes et les hommes sur place qui, en créant des ressources propres via des activités de faible valeur ajoutée mais organisées dans des circuits de proximité, construisent la résilience du territoire dans la durée et offrent des solutions d'avenir. Avant toute révolution agricole ou industrielle, le renforcement des savoirs locaux et l'amélioration des services de base de proximité apparaissent comme une réponse nécessaire et atteignable dans le court terme.

En particulier, des échanges transfrontaliers qui seraient repensés pour profiter davantage au territoire, et qui dynamiseraient des activités agricoles et d'élevage plus complémentaires de part et d'autre de la frontière, font partie des ressources activables. De même, l'exploitation dans une économie verte des réserves naturelles encore préservées dans l'est, qui elle aussi serait pensée dans une logique de coopération transfrontalière, est une piste intéressante.

Les études conduites révèlent et caractérisent les déséquilibres structurels spatiaux du territoire transfrontalier. En comparaison avec les terroirs saturés de l'ouest, bénéficiant des meilleures infrastructures de marché et de connexions aux axes de commerce, des espaces dans l'est sont encore ouverts mais faiblement dotés en infrastructures. La tendance est la conquête progressive par l'agriculture et les villages de la partie est du territoire, avec une pression grandissante sur les ressources naturelles. Mais le désenclavement sans projet économique et territorial global va simplement conduire à reproduire les dynamiques des dernières décennies, et en particulier l'épuisement des ressources et la saturation des terroirs. Résorber durablement les déséquilibres suppose un projet global, valorisant les spécificités de chaque partie du territoire.

Plus largement, les atouts du territoire ne sont pas exceptionnels et remarquables; leur valorisation pour résorber les inégalités territoriales reste soumise à des besoins d'investissement conséquents. L'isolement du territoire dans ses espaces nationaux respectifs constitue à ce titre un frein indéniable, de même que l'inaboutissement de la décentralisation dans chaque pays, qui augmente la dépendance des populations envers des biens, des ressources et des services de base trop distants.

Bien évidemment, la résolution de la crise sécuritaire apparaît enfin comme une condition nécessaire au déploiement d'un projet territorial cohérent. L'insécurité freine les activités économiques, mais aussi creuse les déséquilibres préexistants, en touchant davantage les espaces déjà fragilisés.

La prospective et le diagnostic réalisés, puis la réflexion menée sur les recommandations possibles pour le développement, insistent sur le fait que les espaces transfrontaliers font territoire, ne fonctionnant toutefois que grâce aux populations, 
sans bénéficier d'aucune stratégie politique d'intégration. Une meilleure coopération transfrontalière est un préalable important à tout effort politique de développement des potentiels de ce territoire. Les dimensions politiques et institutionnelles sont donc fondamentales et constituent un prérequis pour penser un développement soutenable du territoire, fondé sur la réappropriation de sa position stratégique dans la région, et sur une revalorisation des actifs naturels de ses espaces les plus fragiles. Avec cette étude participative, les autorités nationales et régionales disposent d'une représentation intégrée inédite des dynamiques réelles, non tronquée conventionnellement par le tracé des frontières, qui de plus exprime des attentes collectives. En ce sens, ce travail se veut être un objet médiateur de dialogue pour la coopération transfrontalière.

\subsection{INSCRIRE LA MOBILITÉ PASTORALE DANS CETTE STRATÉGIE TRANSFRONTALIÈRE}

Les différents défis identifiés permettent par ailleurs d'éclairer le rôle que l'élevage mobile pourrait jouer dans les progrès politiques et stratégiques du territoire et, inversement, les bénéfices que la transhumance pourrait tirer d'une meilleure gouvernance locale et d'une meilleure coopération transfrontalière.

Indéniablement important pour la zone par ses retombées économiques et sa capacité à mobiliser les ressources naturelles encore disponibles, le pastoralisme peut être un sous-secteur clé pour penser la réduction des déséquilibres territoriaux. C'est en effet dans l'est que les couloirs peuvent encore se maintenir et il semble peu probable qu'ils s'ouvrent à nouveau dans les zones très denses de l'ouest.

La transhumance à elle seule n'est pas un projet de territoire, il faut penser les parcours dans leurs possibles concurrences et complémentarités avec d'autres activités et trouver des compromis, accompagner le déplacement des hommes et des animaux en les régulant par rapport à l'ensemble des flux de biens et de marchandises traversant le territoire. Maintenir les mobilités verticales dans l'est peut protéger les espaces naturels; organiser les flux de bétail par des pistes commerciales transversales reconnues peut servir de base à un plan global de circulation des hommes et des biens, pour mieux irriguer toutes les parties du territoire. Une telle stratégie ne peut se concevoir dans une gouvernance cloisonnée entre le Togo et le Burkina Faso.

Par ailleurs, les préjugés trop souvent véhiculés par la presse généraliste sur une transhumance source de tensions et d'insécurité pourraient être dépassés par un débat éclairé sur ses réalités et son fonctionnement très largement paisibles. Plus encore, si elle est encadrée et organisée plutôt que contrainte, si elle bénéficie d'une gouvernance ouverte, transparente et participative, la transhumance peut servir d'exemple pour lutter contre l'insécurité dans la région, en particulier en utilisant mieux le levier d'une meilleure coopération transfrontalière. Organiser la transhumance, c'est aussi renforcer la collaboration et le renseignement au profit de la paix civile. En ce sens, cette étude démontre les effets négatifs du délaissement du secteur productif de l'élevage transhumant dans des régions marginalisées et plaide pour une coopération transfrontalière renforcée. 


\subsection{ENTRETENIR UNE DYNAMIQUE COLLECTIVE TRANSFRONTALIÈRE ET NON CORPORATISTE POUR RENFORCER LA RÉFLEXION STRATÉGIQUE}

Quelques éléments techniques inhérents à l'étude restent à réaliser. Ainsi, le présent rapport devra être discuté, approprié, amélioré et valoriser les «experts du territoire» ayant participé aux ateliers et leurs institutions d'appartenance par d'autres groupes stratégiques qui n'étaient pas impliqués. Mais au-delà, le plaidoyer, déjà entamé dans le processus de l'étude, reste à mener auprès des autorités locales et nationales, des services techniques centralisés et déconcentrés, ainsi que des principaux acteurs de la société civile. Cet effort de partage, au-delà de la diffusion de la connaissance produite, pourrait être une étape pour «embarquer» d'autres acteurs dans le développement local transfrontalier et dans une approche territoriale et anticipatrice d'une transhumance apaisée.

Deux courts films d'animation, décrivant la méthode de la prospective territoriale et présentant quelques-uns des scénarios, sont disponibles et utilisables à la demande par l'ensemble des partenaires intéressés (ici et là). D'autres supports, notamment des frises dessinées présentant les scénarios facilement manipulables, sont rassemblés dans un coffret. Films et dessins permettent, dans un langage et une forme accessibles, d'introduire les grands principes travaillés ici et de les utiliser pour l'action.

La prospective et le diagnostic réalisés doivent être considérés comme des points de départ d'une démarche plus longue, mais qui n’appartient plus aux équipes de recherche, et doit être prise en charge par les acteurs locaux et nationaux du développement, chacun dans ses compétences. Les premières propositions et recommandations sur la gouvernance et la coopération transfrontalières ne sont que des ébauches, qui plus est dans un champ thématique réduit. Il conviendrait de poursuivre ce premier travail, en abordant d'autres domaines définis par les variables motrices. Le diagnostic territorial, les projections et les scénarios pourraient ainsi être utilisés pour débattre des actions et recommandations sur le renforcement du capital humain, la sécurité, la natalité, la structuration du monde professionnel, les activités minières et la préservation des écosystèmes.

Puis, pour les recommandations sur la coopération et la gouvernance transfrontalière comme pour les forces motrices identifiées, un travail de précision, via des études et des ateliers supplémentaires, permettrait d'envisager leur mise en ouvre. Il conviendrait ici de travailler le cadre institutionnel et réglementaire de cette mise en œuvre, et la cohérence et compatibilité entre l'action publique aux différentes échelles, avec un point d'attention sur la décentralisation. Comme pour l'étape précédente, ce travail ne relève pas des compétences de la recherche mais d'une action citoyenne.

Enfin, le partage des travaux au niveau régional est une priorité. La méthode et ses grands résultats doivent être présentés et discutés, avec des partenaires des territoires et du pastoralisme, au Togo et au Burkina, mais aussi possiblement dans d'autres territoires transfrontaliers stratégiques. Ainsi le nord du Bénin pourrait être utilement impliqué dans des prospectives territoriales.

Plus largement, les résultats produits par la prospective territoriale transfrontalière pourraient accompagner la réflexion de la conférence interministérielle sur la transhumance apaisée, voire d'autres instances sous régionales. Cet effort de dissémination relève de la recherche, mais bien entendu plus encore des acteurs locaux qui ont été formés à l'anticipation dans le cadre de cette étude et de toute personne informée susceptible d'en utiliser les enseignements. 



\section{Bibliographie indicative}

Bahan, D. 2009. Recensement général de la population et de l'habitation de 2006 (RGPH-2006). Monographie de la région de l'Est. INSD. Ouagadougou.

Baya, B., Zida, H. et Bonkoungou, Z. 2009. Recensement général de la population et de l'habitation de 2006 (RGPH-2006): analyse des résultats définitifs. Thème 7: Mortalité. Page 54. INSD. Ouagadougou.

Blundo Canto, G., Barret D., Faure G., Hainzelin, E., Monier C., Triomphe B., Vall E. (illus.). 2018. ImpresS ex ante. Une proposition de démarche pour construire ex ante les chemins de l'impact. Cirad. Montpellier, France

Bourgeois, R. et Jésus, F. 2004. Participatory prospective analysis: exploring and anticipating challenges with stakeholders. CAPSA Monograph 46. UNESCAPCAPSA. Bogor, Indonésie.

Bourgeois, R., Penunia, E., Bisht, S. et Boruk, D. 2017. Foresight for all: Coelaborative scenario building and empowerment. Technological Forecasting and Social Change, 124 (2017),178-188. https://doi.org/10.1016/j.techfore.2017.04.018

Conseil régional du Centre-Est. 2018. Plan régional de développement du CentreEst (2018-2022). Ministère de l'administration territoriale et de la décentralisation. Ouagadougou.

Conseil régional de l'Est. 2018. Plan régional de développement, rapport de diagnostic. Ministère de l'administration territoriale et de la décentralisation. Ouagadougou.

Cherbib, H. 2018. Le djihadisme au Sahel: exploiter les instabilités locales. Annuaire IEMed. de la Méditerranée. Sécurité et politiques. Paris. https://www.iemed. org/observatori/arees-danalisi/arxius-adjunts/anuari/med.2018/fr/Djihadisme_ Sahel Cherbib medyearbook2018 fr.pdf

Dabire, B. et Kone, H. 2009. Recensement général de la population et de l'habitation de 2006 (RGPH-2006). Analyse des résultats définitifs. Thème 8: Migrations. INSD. Ouagadougou.

DGSCN. Direction générale de la statistique et de la comptabilité nationale. 2010. Quatrième recensement général de la population et de l'habitat - novembre 2010. Publication des résultats définitifs: volume spécial. Lomé.

DGSCN. Direction générale de la statistique et de la comptabilité nationale. 2011. Recensement général de la population et de l'habitat (06 au 21 novembre 2010). Résultats définitifs. Lomé.

Dreborg K. H. 1996. Essence of backcasting. Futures, Vol. 28, No. 9, pp. 813-828. https://www.unifg.it/sites/default/files/allegatiparagrafo/07-07-2014/essenceof-backcasting 1996 futures.pdf

Godet, M., Durance, P. 2008. La prospective stratégique. Pour les entreprises et les territoires. Collection Topos, Dunod. Paris.

Gozo, K. A. 1989. Composition et distribution géographique de la population togolaise. République togolaise. Lomé.

Guengant, J. P., Lankoande, M., Tapsoba, T. V. M. E. et Zanou, B. 2009. Recensement général de la population et de l'habitation de 2006 (RGPH-2006): Projections démographiques 2007-2050. INSD. Ouagadougou. 
INSD. Institut national de la statistique et de la démographie. 2000. Analyse des résultats du recensement général de la population et de l'habitation de 1996: volume II. Ouagadougou.

INSD. Institut national de la statistique et de la démographie. 2008. Recensement général de la population et de l'habitation de 2006. Résultats définitifs. Ouagadougou.

INSD. Institut national de la statistique et de la démographie. 2009. Projections démographiques de 2007 à 2020 par région et province. Ouagadougou.

INSD. Institut national de la statistique et de la démographie. 2011. La région du Centre-Est en chiffres. Ouagadougou.

Koupogbe, E. S. et Tete, K. G. 2015. Perspectives démographiques du Togo 2011-2031. INSEED/Togo

Losch, B. 2016. Structural transformation to boost youth labour demand in subSaharan Africa: The role of agriculture, rural areas and territorial development. Employment Working Papers No.204. OIT. Genève. http://www.ilo.org/ employment/Whatwedo/Publications/working-papers/WCMS 533993/lang-en/index.htm

Loye, A. S. et Gouem, M.S. 2009. Recensement général de la population et de l'habitation de 2006 (RGPH-2006). Monographie de la région du Centre-Est. INSD. Ouagadougou.

Ministère de la santé et de la protection sociale. République togolaise. 2016. Plan national de développement sanitaire 2017-2022. Lomé.

Ouedraogo, M. et Ripama, T. 2009. Recensement général de la population et de l'habitation (RGPH) de 2006. Analyse des résultats définitifs: état et structure de la population. INSD Ouagadougou.

République togolaise. 2012. Plan national de développement sanitaire du Togo 2012-2015. Lomé.

Sangli, G. 2011. La dynamique du peuplement dans le sud-ouest du Burkina Faso de 1975 à 2006. Essai à partir des données de recensement des localités. Thèse de doctorat de géographie. Université Paris 1 Panthéon-Sorbonne. Paris.

Sourisseau, J.M., Bougnoux, N., Bélières, J.F., Bourgeois, R., Soumaré, M., Rasolofo, P. 2017. Imaginer l'avenir des territoires pour relever les défis de la démographie et de l'emploi en Afrique subsaharienne. Perspective - Cirad (44): p. 1-4. Montpellier, France. http://dx.doi.org/10.19182/agritrop/00002. http://www.cirad.fr/publications-ressources/science-pour-tous/\%28type\%29/ perspective-policy-brief

Sourisseau J.M., Bélières J.F., Bourgeois R., Soumaré M., Rasolofo P., Guengant J.P., Bougnoux N., Losch B., Ramanitriniony H.K., Coulibaly B., Traoré S., Razafimiarantsoa T.T., Andrianantoandro V.T., Ramarijaona M., Burnod P. 2017. Penser ensemble l'avenir d'un territoire. Diagnostic et prospective territoriale au Mali et à Madagascar (Études de l'AFD). AFD. Paris. https://www.afd.fr/index.php/fr/penser-ensemble-avenir-territoire

Tete, K. G. et Djibom, K. A. 2016. Natalité et fécondité, INSEED/Togo. Lomé.

URD. Unité de recherche démographique. 2006. Peuplement, mobilité et développement dans un milieu défavorisé: le cas de la région des Savanes au Togo. Rapport de recherche. DGSCN. Lomé. 


\section{COLLECTION EN LIGNE}

\section{DOCUMENTS DE TRAVAIL FAO PRODUCTION ET SANTÉ ANIMALES}

1. The use of cash transfers in livestock emergencies and their incorporation into Livestock Emergency Guidelines and Standards (LEGS), 2011 (En) http://www.fao.org/3/a-i2256e.pdf

2. Mapping supply and demand for animal-source foods to 2030, 2011 (En) http://www.fao.org/3/a-i2425e.pdf

3. Notes on Livestock, Food Security and Gender Equity, 2011 (En) http://www.fao.org/3/a-i2426e.pdf

4. Wealth Index mapping in the Horn of Africa, 2011 (En) http://www.fao.org/3/a-i2427e.pdf

5. Évolution du secteur avicole en Tunisie, 2011 (Fr) http://www.fao.org/3/a-i2549f.pdf

6. Status of animal nutrition research and development activities in Tajikistan, Kyrgyzstan and Azerbaijan, 2012 (En, Ru) http://www.fao.org/3/a-i2582e.pdf - http://www.fao.org/3/a-i2582r.pdf

7. An assessment of the socio-economic impacts of global rinderpest eradication - Methodological issues and applications to rinderpest control programmes in Chad and India, 2012 (En) http://www.fao.org/3/a-i2584e.pdf

8. Use of lesser-known plants and plant parts as animal feed resources in tropical regions, 2012 (En) http://www.fao.org/3/a-i2629e.pdf

9. Poverty mapping in Uganda - Extrapolating household expenditure data using environmental data and regression techniques, 2012 (En) http://www.fao.org/3/i2705e/i2705e.pdf

10. How can animal health systems support small-scale poultry producers and traders? - Reflections on experience with HPAl, 2012 (En) http://www.fao.org/3/a-i2739e.pdf

11. Mapping Influenza A (H5N1) Virus Transmission Pathways and Critical Control Points in Egypt, 2013 (En) http://www.fao.org/3/a-i3272e.pdf

12. Développement de l'aviculture familiale - Enjeux, opportunités et contraintes, 2014 (En, Fr) http://www.fao.org/3/a-i3595e.pdf - http://www.fao.org/3/a-i3595f.pdf

13. Impact of mastitis in small scale dairy production systems, 2014 (En) http://www.fao.org/3/a-i3377e.pdf

14. Comparative performance of Sonali chickens, commercial broilers, layers and local non-descript (deshi) chickens in selected areas of Bangladesh, 2015 (En) http://www.fao.org/3/a-i4725e.pdf

15. Identification of indicators for evaluating of sustainable animal diets, 2016 (En) http://www.fao.org/3/a-i5200e.pdf

16. Creating employment potential in small-ruminant value chains in the Ethiopian Highlands, 2017 (En) http://www.fao.org/3/a-i6906e.pdf

Disponibilité: Décembre 2021
Ar - Arabe
En - Anglais
Es - Espagnol
$\mathrm{Fr}$ - Français
Ru - Russe
Zh - Chinois

Multilingue

** En préparation 




Cette synthèse présente la méthode et les résultats d'une recherche participative menée de chaque côté de la frontière entre le Burkina Faso et le Togo, où les questions de transhumance sont intégrées dans une prospective sur le territoire transfrontalier. Les crises affectant le pastoralisme dans la région subsaharienne depuis quelques années s'inscrivent dans des conflits territoriaux. La méthode innovante de prospective territoriale permet aux acteurs locaux et aux décideurs de replacer le pastoralisme dans le développement transfrontalier et de s'extraire des tensions actuelles en imaginant différents futurs pour leur territoire. Deux questions ont été posées: quels pourraient être les futurs du territoire transfrontalier entre Togo et Burkina Faso en 2035? Quels rôles et quelles fonctions pourrait jouer le pastoralisme dans ces futurs?

Le document offre un diagnostic territorial, fondé sur 30 ans de données statistiques, 61 interviews de personnes ressources du territoire et une enquête auprès de 568 personnes dans 239 familles tirées au sort des deux côtés de la frontière. Ce diagnostic a nourri trois ateliers participatifs durant lesquels les acteurs locaux ont identifié et hiérarchisé les forces motrices des transformations. Ils ont construit huit scénarios, de «l'idéal» à la «crise du terroir» en passant par "les sentiers du désert», puis ont réintroduit la transhumance transfrontalière dans ces images du futur.

La méthode innovante de prospective territoriale a offert des espaces de concertation originaux et inédits à des experts locaux représentatifs de plusieurs facettes de la population du territoire transfrontalier qui sont désormais formés à utiliser cet outil. Ces experts ont commencé à formuler des propositions d'actions concrètes pour une transhumance apaisée, en se centrant sur l'amélioration de la coopération transfrontalière, la gouvernance locale et la sécurité. Ils informent et enrichissent ainsi leurs plaidoyers civils et les concertations sur les schémas d'aménagement régional. La réflexion et les recommandations sur le développement territorial en conclusion du document renforcent les places et rôles de la transhumance, favorisant sa coexistence apaisée avec les autres secteurs d'activité dans les territoires. 